\title{
Two-body hadronic weak decays of antitriplet charmed baryons
}

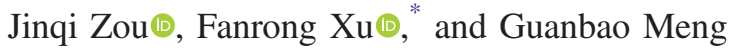 \\ Department of Physics, Jinan University, Guangzhou 510632, People's Republic of China \\ Hai-Yang Cheng (0) \\ Institute of Physics, Academia Sinica, Taipei, Taiwan 115, Republic of China
}

(Received 31 October 2019; published 14 January 2020; corrected 29 January 2021)

\begin{abstract}
We study Cabibbo-favored (CF) and singly Cabibbo-suppressed (SCS) two-body hadronic weak decays of the antitriplet charmed baryons $\Lambda_{c}^{+}, \Xi_{c}^{0}$, and $\Xi_{c}^{+}$with more focus on the last two. Both factorizable and nonfactorizable contributions are considered in the topologic diagram approach. The estimation of nonfactorizable contributions from $W$-exchange and inner $W$-emission diagrams relies on the pole model and current algebra. The nonperturbative parameters in both factorizable and nonfactorizable parts are calculated in the MIT bag model. Branching fractions and up-down decay asymmetries for all the CF and SCS decays of antitriplet charmed baryons are presented. The prediction of $\mathcal{B}\left(\Xi_{c}^{+} \rightarrow \Xi^{0} \pi^{+}\right)$agrees well with the measurements inferred from Belle and CLEO, while the calculated $\mathcal{B}\left(\Xi_{c}^{0} \rightarrow \Xi^{-} \pi^{+}\right)$is too large compared to the recent Belle measurement. We conclude that these two $\Xi_{c} \rightarrow \Xi \pi^{+}$modes cannot be simultaneously explained within the current-algebra framework for $S$-wave amplitudes. This issue needs to be resolved in future study. The long-standing puzzle with the branching fraction and decay asymmetry of $\Lambda_{c}^{+} \rightarrow \Xi^{0} K^{+}$is resolved by noting that only the type-II $W$-exchange diagram contributes to this mode. We find that not only does the calculated rate agree with experiment but also the predicted decay asymmetry is consistent with the SU(3)-flavor symmetry approach in sign and magnitude. Likewise, the CF mode $\Xi_{c}^{0} \rightarrow \Sigma^{+} K^{-}$and the SCS decays $\Xi_{c}^{0} \rightarrow p K^{-}, \Sigma^{+} \pi^{-}$proceed only through type-II $W$ exchange. They are predicted to have large and positive decay asymmetries. These features can be tested in the near future.
\end{abstract}

DOI: $10.1103 /$ PhysRevD.101.014011

\section{INTRODUCTION}

Recently, there has been significant progress in the experimental study of charm physics. In the meson sector, LHCb measured the $C P$ asymmetry difference between $D^{0} \rightarrow K^{-} K^{+}$and $D^{0} \rightarrow \pi^{-} \pi^{+}$, giving $\Delta A_{\mathrm{CP}}=(-15.4 \pm$ 2.9) $\times 10^{-4}$ [1], which is the first observation of $C P$ violation in the charm sector. The progress in charmed baryon physics is also impressive. The long-quested doubly charmed baryon was first observed through the process $\Xi_{c c}^{++} \rightarrow \Lambda_{c}^{+} K^{-} \pi^{+} \pi^{+}$at LHCb in 2017 [2]. Later in 2018, the lifetime of $\Xi_{c c}^{++}$[3], its mass and the two-body weak decay channel $\Xi_{c c}^{++} \rightarrow \Xi_{c}^{+} \pi^{+}$[4], were measured by LHCb. Some breakthrough has also been made in singly charmed baryons as well, especially the lightest one, $\Lambda_{c}^{+}$. Both Belle [5] and BESIII [6] have measured the absolute branching fraction of the decay $\Lambda_{c}^{+} \rightarrow p K^{-} \pi^{+}$. A new average of

\footnotetext{
*fanrongxu@jnu.edu.cn
}

Published by the American Physical Society under the terms of the Creative Commons Attribution 4.0 International license. Further distribution of this work must maintain attribution to the author(s) and the published article's title, journal citation, and DOI. Funded by SCOAP.
$(6.28 \pm 0.32) \%$ for this benchmark mode is quoted by the Particle Data Group (PDG) [7]. The measurement of $\Lambda_{c}^{+} \rightarrow p \pi^{0}, p \eta[8]$ indicated that singly Cabibbosuppressed (SCS) decays are ready to access.

In addition to $\Lambda_{c}^{+}$, there have been some new developments in the study of $\Xi_{c}^{0}$ and $\Xi_{c}^{+}$, the other two singly charmed baryons in the antitriplet. By using a data set comprising $(772 \pm 11) \times 10^{6} B \bar{B}$ pairs collected at $\Upsilon(4 S)$ resonance, Belle was able to measure the absolute branching fraction for $B^{-} \rightarrow \bar{\Lambda}_{c}^{-} \Xi_{c}^{0}$ [9]. Combining the subsequently measured product branching fractions such as $\mathcal{B}\left(B^{-} \rightarrow \bar{\Lambda}_{c}^{-} \Xi_{c}^{0}\right) \mathcal{B}\left(\Xi_{c}^{0} \rightarrow \Xi^{-} \pi^{+}\right)$, Belle reported the first weak decay of $\Xi_{c}^{0}[9]$,

$$
\mathcal{B}\left(\Xi_{c}^{0} \rightarrow \Xi^{-} \pi^{+}\right)=(1.80 \pm 0.50 \pm 0.14) \times 10^{-2} .
$$

Using the same technique, a channel of two-body weak decay with a vector meson in final state was also measured, $\mathcal{B}\left(\Xi_{c}^{+} \rightarrow p \bar{K}^{0 *}(892)\right)=(0.25 \pm 0.16 \pm 0.04) \times 10^{-2}[10]$. It is worth pointing out that the absolute branching fraction for three-body decay was obtained by Belle [10] to be $\mathcal{B}\left(\Xi_{c}^{+} \rightarrow \Xi^{-} \pi^{+} \pi^{+}\right)=(2.86 \pm 1.21 \pm 0.38) \times 10^{-2}$, from which we can read 
TABLE I. Branching fractions (upper entry) and up-down decay asymmetries $\alpha$ (lower entry) of Cabibbo-allowed $\Xi_{c}^{+, 0} \rightarrow \mathcal{B}+P$ decays in various early model calculations. All the model results for branching fractions (in percent) have been normalized using the current world averages of $\tau\left(\Xi_{c}^{+}\right)$and $\left.\tau_{(} \Xi_{c}^{0}\right)$ [see Eq. (3) below].

\begin{tabular}{|c|c|c|c|c|c|c|c|c|}
\hline \multirow[b]{2}{*}{ Decay } & \multirow{2}{*}{$\begin{array}{c}\text { Körner, } \\
\text { Krämer [20] }\end{array}$} & \multirow{2}{*}{$\begin{array}{c}\text { Xu, } \\
\text { Kamal [22] }\end{array}$} & \multicolumn{2}{|c|}{ Cheng, Tseng [23] } & \multirow{2}{*}{$\begin{array}{c}\text { Ivanov et al. } \\
{[21]}\end{array}$} & \multirow{2}{*}{$\begin{array}{c}\text { Żenczykowski } \\
{[24]}\end{array}$} & \multirow{2}{*}{$\begin{array}{c}\text { Sharma, } \\
\text { Verma [25] }\end{array}$} & \multirow{2}{*}{$\begin{array}{c}\text { Experiment } \\
{[7,9]}\end{array}$} \\
\hline & & & $\mathrm{CA}$ & Pole & & & & \\
\hline$\Xi_{c}^{+} \rightarrow \Sigma^{+} \bar{K}^{0}$ & 6.66 & 0.46 & 0.05 & 0.87 & 4.05 & & & \\
\hline$\Xi_{c}^{+} \rightarrow \Xi^{0} \pi^{+}$ & 3.65 & 3.47 & 0.87 & 4.06 & 5.78 & & & $1.57 \pm 0.83$ \\
\hline$\Xi_{c}^{0} \rightarrow \Lambda \bar{K}^{0}$ & 0.17 & 0.50 & 1.36 & 0.37 & 0.55 & & & \\
\hline$\Xi_{c}^{0} \rightarrow \Sigma^{0} \bar{K}^{0}$ & 1.61 & 0.14 & 0.03 & 0.18 & 0.26 & & & \\
\hline$\Xi_{c}^{0} \rightarrow \Sigma^{+} K^{-}$ & 0.17 & 0.17 & & & 0.35 & & & \\
\hline$\Xi_{c}^{0} \rightarrow \Xi^{0} \pi^{0}$ & 0.05 & 0.77 & 1.71 & 0.38 & 0.05 & & & \\
\hline$\Xi_{c}^{0} \rightarrow \Xi^{-} \pi^{+}$ & 1.42 & 2.37 & 1.13 & 1.71 & 1.60 & & & $1.80 \pm 0.52$ \\
\hline$\Xi_{c}^{0} \rightarrow \Xi^{0} \eta$ & 0.32 & & & & 0.37 & & & \\
\hline$\Xi_{c}^{0} \rightarrow \Xi^{0} \eta^{\prime}$ & 1.16 & & & & 0.41 & & & \\
\hline$\Xi_{c}^{+} \rightarrow \Sigma^{+} \bar{K}^{0}$ & -1.0 & 0.24 & 0.43 & -0.09 & -0.99 & 1.0 & 0.54 & \\
\hline$\Xi_{c}^{+} \rightarrow \Xi^{0} \pi^{+}$ & -0.78 & -0.81 & -0.77 & -0.77 & -0.97 & 1.0 & -0.27 & \\
\hline$\Xi_{c}^{0} \rightarrow \Lambda \bar{K}^{0}$ & -0.76 & 1.00 & -0.88 & -0.73 & -0.75 & -0.29 & -0.79 & \\
\hline$\Xi_{c}^{0} \rightarrow \Sigma^{0} \bar{K}^{0}$ & -0.96 & -0.99 & 0.85 & -0.59 & -0.55 & -0.50 & 0.48 & \\
\hline$\Xi_{c}^{0} \rightarrow \Sigma^{+} K^{-}$ & 0 & 0 & & & 0 & 0 & 0 & \\
\hline$\Xi_{c}^{0} \rightarrow \Xi^{0} \pi^{0}$ & 0.92 & 0.92 & -0.78 & -0.54 & 0.94 & 0.21 & -0.80 & \\
\hline$\Xi_{c}^{0} \rightarrow \Xi^{-} \pi^{+}$ & -0.38 & -0.38 & -0.47 & -0.99 & -0.84 & -0.79 & -0.97 & $-0.6 \pm 0.4$ \\
\hline$\Xi_{c}^{0} \rightarrow \Xi^{0} \eta$ & -0.92 & & & & -1.0 & 0.21 & -0.37 & \\
\hline$\Xi_{c}^{0} \rightarrow \Xi^{0} \eta^{\prime}$ & -0.38 & & & & -0.32 & -0.04 & 0.56 & \\
\hline
\end{tabular}

$$
\mathcal{B}\left(\Xi_{c}^{+} \rightarrow \Xi^{0} \pi^{+}\right)=(1.57 \pm 0.83) \%,
$$

where use of $\Gamma\left(\Xi_{c}^{+} \rightarrow \Xi^{0} \pi^{+}\right) / \Gamma\left(\Xi_{c}^{+} \rightarrow \Xi^{-} \pi^{+} \pi^{+}\right)=(0.55 \pm$ $0.13 \pm 0.09)$ obtained by the CLEO [11] has been made.

For lifetimes of the antitriplet charmed baryons, we quote the new world averages (in units of $10^{-13} \mathrm{~s}$ ),

$$
\begin{aligned}
\tau\left(\Lambda_{c}^{+}\right) & =2.03 \pm 0.02, \quad \tau\left(\Xi_{c}^{+}\right)=4.56 \pm 0.05, \\
\tau\left(\Xi_{c}^{0}\right) & =1.53 \pm 0.02,
\end{aligned}
$$

dominated by the most recent lifetime measurements by the $\mathrm{LHCb}$ [12]. Note that the measured $\Xi_{c}^{0}$ lifetime by the LHCb is approximately 3.3 standard deviations larger than the old world average value [7].

Inspired by latest experimental results of $\Xi_{c}$ decays, there have been some efforts from theorists [13-18]. Indeed, the study of charmed baryon weak decays, including the charged and neutral $\Xi_{c}$ baryons, is an old subject. To understand the underlying dynamical mechanism in hadronic weak decays, one may draw the topological diagrams according to the hadron's content [19]. In charmed baryon decays, nonfactorizable contributions from $W$-exchange or inner $W$-emission diagrams play an essential role and they cannot be neglected, in contrast with the negligible effects in heavy meson decays. The fact that all the decays of $\Xi_{c}^{+, 0}$ receive nonfactorizable contributions, especially some decays such as $\Xi_{c}^{0} \rightarrow \Sigma^{+} K^{-}, \Xi^{0} \pi^{0}$, proceed only through purely nonfactorizable diagrams allows us to check the importance and necessity of nonfactorizable contributions. However, so far we still do not have a reliable phenomenological model to calculate charmed baryon hadronic decays. In the 1990 s various techniques were developed, including relativistic quark model [20,21], pole model [22-24], and current algebra [23,25], to estimate the nonfactorizable effects in Cabbibo-favored $\Xi_{c}^{+, 0}$ decays. The predicted branching fractions and decay asymmetries in various early model calculations are summarized in Table I. ${ }^{1}$

Now that with more experimental data accumulated, there are some updated studies in theory [13-17]. In these works except [17], the experimental results are taken as input and global fitting analyses are carried out at the hadron level based on SU(3) flavor symmetry without resorting to the detailed dynamics. Apparently, a reconsideration of charmed baryon weak decays, revealing the dynamics at the quark level, is timely and necessary. The pole model is one of the choices.

\footnotetext{
${ }^{1}$ For early model calculations of Cabibbo-allowed $\Lambda_{c}^{+} \rightarrow \mathcal{B}+P$ decays, see Table I of [26].
} 
In the pole model, important low-lying $1 / 2^{+}$and $1 / 2^{-}$ states are usually considered under the pole approximation. In the decay with a pseudoscalar in the final state, $\mathcal{B}_{c} \rightarrow \mathcal{B}+P$, the nonfactorizable $S$ - and $P$-wave amplitudes are dominated by $1 / 2^{-}$low-lying baryon resonances and $1 / 2^{+}$ground state baryons, respectively. The $S$-wave amplitude can be further reduced to current algebra in the soft-pseudoscalar limit. That is, the evaluation of the $S$-wave amplitude does not require the information of the troublesome negative-parity baryon resonances that are not well understood in the quark model. The methodology was developed and applied in the earlier work [23]. It turns out that if the $S$-wave amplitude is evaluated in the pole model or in the covariant quark model and its variant, the decay asymmetries for both $\Lambda_{c}^{+} \rightarrow \Sigma^{+} \pi^{0}$ and $\Sigma^{0} \pi^{+}$are always predicted to be positive, while it was measured to be $-0.45 \pm 0.31 \pm 0.06$ for $\Sigma^{+} \pi^{0}$ by CLEO [27]. In contrast, current algebra always leads to a negative decay asymmetry for the aforementioned two modes: -0.49 in [23], -0.31 in [25], -0.76 in [28] and -0.47 in [29]. The issue with the sign of $\alpha\left(\Lambda_{c}^{+} \rightarrow \Sigma^{+} \pi^{0}\right)$ was finally resolved by BESIII. The decay asymmetry parameters of $\Lambda_{c}^{+} \rightarrow \Lambda \pi^{+}, \Sigma^{0} \pi^{+}, \Sigma^{+} \pi^{0}$, and $p K_{S}$ were recently measured by BESIII [30] (see Table III below), for example, $\alpha\left(\Lambda_{c}^{+} \rightarrow \Sigma^{+} \pi^{0}\right)=-0.57 \pm$ 0.12 was obtained. Hence, the negative sign of $\alpha\left(\Lambda_{c}^{+} \rightarrow\right.$ $\Sigma^{+} \pi^{0}$ ) measured by CLEO is nicely confirmed by BESIII. This is one of the strong reasons why we adapt current algebra to work out parity-violating amplitudes.

It is well known that there is a long-standing puzzle with the branching fraction and decay asymmetry of $\Lambda_{c}^{+} \rightarrow \Xi^{0} K^{+}$. The calculated branching fraction turns out to be too small compared to experiment and the decay asymmetry is predicted to be 0 owing to the vanishing $S$ wave amplitude. We examine this issue in this work and point out a solution to this puzzle. This has important implications to the $\Xi_{c}^{0}$ sector where the CF mode $\Xi_{c}^{0} \rightarrow$ $\Sigma^{+} K^{-}$and the SCS decays $\Xi_{c}^{0} \rightarrow p K^{-}, \Sigma^{+} \pi^{-}$encounter similar problems in naive calculations.

Recently, we have followed this approach to calculate SCS decays of $\Lambda_{c}^{+}$[26], in which the predictions of $\Lambda_{c}^{+} \rightarrow p \pi^{0}, p \eta$ are in good agreement with the BESIII measurement. In this work, we continue working in the pole model together with current algebra to compute both CF and SCS two-body weak decays of $\Xi_{c}$ baryons.

In short, this work is motivated mainly by three parts: (i) new data on the branching fractions and lifetimes of $\Xi_{c}^{+, 0}$, (ii) correct sign predictions of $\alpha$ in $\Lambda_{c}^{+} \rightarrow \Sigma^{+} \pi^{0}$ and $\Sigma^{0} \pi^{+}$by current algebra, and (iii) the long-standing puzzle of $\Lambda_{c}^{+} \rightarrow \Xi^{0} K^{+}$and its implication to the $\Xi_{c}$ sector.

This paper is organized as follows. In Sec. II we set up the formalism for computing branching fractions and updown decay asymmetries, including contributions from both factorizable and nonfactorizable terms. Numerical results are presented in Sec. III. A conclusion is given in
Sec. IV. In Appendix A, we write down the baryon wave functions to fix our convention and then examine their behavior under $U, V$, and $I$ spin in Appendix B. Appendix $\mathrm{C}$ is devoted to the form factors for $\Lambda_{c}^{+} \rightarrow \mathcal{B}$ transitions evaluated in the MIT bag model. The expressions of baryon matrix elements and axial-vector form factor calculated in the MIT bag model are presented in Appendix D.

\section{FORMALISM}

\section{A. Kinematics}

Without loss of generality, the amplitude for the twobody weak decay $\mathcal{B}_{i} \rightarrow \mathcal{B}_{f} P$ can be parametrized as

$$
M\left(\mathcal{B}_{i} \rightarrow \mathcal{B}_{f} P\right)=i \bar{u}_{f}\left(A-B \gamma_{5}\right) u_{i}
$$

where $P$ denotes a pseudoscalar meson. Based on the $S$ - and $P$-wave amplitudes, $A$ and $B$, the decay width and up-down spin asymmetry are given by

$$
\begin{gathered}
\Gamma=\frac{p_{c}}{8 \pi}\left[\frac{\left(m_{i}+m_{f}\right)^{2}-m_{P}^{2}}{m_{i}^{2}}|A|^{2}+\frac{\left(m_{i}-m_{f}\right)^{2}-m_{P}^{2}}{m_{i}^{2}}|B|^{2}\right], \\
\alpha=\frac{2 \kappa \operatorname{Re}\left(A^{*} B\right)}{|A|^{2}+\kappa^{2}|B|^{2}},
\end{gathered}
$$

with $\kappa=p_{c} /\left(E_{f}+m_{f}\right)=\sqrt{\left(E_{f}-m_{f}\right) /\left(E_{f}+m_{f}\right)}$, and $p_{c}$ is the three-momentum in the rest frame of the mother particle.

The $S$ - and $P$-wave amplitudes of the two-body decay generically receive both factorizable and nonfactorizable contributions,

$$
A=A^{\mathrm{fac}}+A^{\mathrm{nf}}, \quad B=B^{\mathrm{fac}}+B^{\mathrm{nf}} .
$$

We should keep in mind that the above formal decomposition is process dependent; not all the processes contain both contributions shown in Eq. (7). To identify the explicit components, one way is to resort to the topological diagram method. In the topological diagram approach, the external $W$ emission and internal $W$ emission from the external quark are usually classified as factorization contributions, while the nonfactorizable contributions arise from inner $W$-emission and $W$-exchange diagrams. Contrary to weak decays of $\Lambda_{c}^{+}$, decay modes proceeding only through factorizable contributions cannot be found in $\Xi_{c}^{+, 0}$ decays.

\section{B. Factorizable contribution}

The description of the factorizable contribution of the charmed baryon decay $\mathcal{B}_{c} \rightarrow \mathcal{B P}$ is based on the effective Hamiltonian approach. 


\section{General expression of factorizable amplitudes}

The effective Hamiltonian for CF process is

$$
\mathcal{H}_{\text {eff }}=\frac{G_{F}}{\sqrt{2}} V_{c s} V_{u d}^{*}\left(c_{1} O_{1}+c_{2} O_{2}\right)+\text { H.c. },
$$

where the four-quark operators are given by

$$
O_{1}=(\bar{s} c)(\bar{u} d), \quad O_{2}=(\bar{u} c)(\bar{s} d),
$$

with $\left(\bar{q}_{1} q_{2}\right) \equiv \bar{q}_{1} \gamma_{\mu}\left(1-\gamma_{5}\right) q_{2}$. The Wilson coefficients to the leading order are given as $c_{1}=1.346$ and $c_{2}=-0.636$ at $\mu=1.25 \mathrm{GeV}$ and $\Lambda_{\mathrm{MS}}^{(4)}=325 \mathrm{MeV}$ [31]. Under naive factorization the amplitude can be written down as

$$
\begin{aligned}
M & =\left\langle P \mathcal{B}\left|\mathcal{H}_{\mathrm{eff}}\right| \mathcal{B}_{c}\right\rangle \\
& =\left\{\begin{array}{l}
\frac{G_{F}}{\sqrt{2}} V_{c s} V_{u d}^{*} a_{2}\langle P|(\bar{s} d)| 0\rangle\left\langle\mathcal{B}|(\bar{u} c)| \mathcal{B}_{c}\right\rangle, \quad P=\bar{K}^{0}, \\
\frac{G_{F}}{\sqrt{2}} V_{c s} V_{u d}^{*} a_{1}\langle P|(\bar{u} d)| 0\rangle\left\langle\mathcal{B}|(\bar{s} c)| \mathcal{B}_{c}\right\rangle, P=\pi^{+},
\end{array}\right.
\end{aligned}
$$

where $a_{1}=c_{1}+\frac{c_{2}}{N}$ and $a_{2}=c_{2}+\frac{c_{1}}{N}$. In terms of the decay constants ${ }^{2}$

$$
\begin{aligned}
\left\langle K(q)\left|\bar{s} \gamma_{\mu}\left(1-\gamma_{5}\right) d\right| 0\right\rangle & =i f_{K} q_{\mu}, \\
\left\langle\pi(q)\left|\bar{u} \gamma_{\mu}\left(1-\gamma_{5}\right) d\right| 0\right\rangle & =i f_{\pi} q_{\mu},
\end{aligned}
$$

and the form factors defined by

$$
\begin{aligned}
& \left\langle\mathcal{B}\left(p_{2}\right)\left|\bar{c} \gamma_{\mu}\left(1-\gamma_{5}\right) u\right| \mathcal{B}_{c}\left(p_{1}\right)\right\rangle \\
& =\bar{u}_{2}\left[f_{1}\left(q^{2}\right) \gamma_{\mu}-f_{2}\left(q^{2}\right) i \sigma_{\mu \nu} \frac{q^{\nu}}{M}+f_{3}\left(q^{2}\right) \frac{q_{\mu}}{M}\right. \\
& \left.\quad-\left(g_{1}\left(q^{2}\right) \gamma_{\mu}-g_{2}\left(q^{2}\right) i \sigma_{\mu \nu} \frac{q^{\nu}}{M}+g_{3}\left(q^{2}\right) \frac{q_{\mu}}{M}\right) \gamma_{5}\right] u_{1},
\end{aligned}
$$

with the momentum transfer $q=p_{1}-p_{2}$, we obtain the amplitude

$$
\begin{aligned}
M\left(\mathcal{B}_{c} \rightarrow \mathcal{B P}\right)= & i \frac{G_{F}}{\sqrt{2}} a_{1,2} V_{u d}^{*} V_{c s} f_{P} \bar{u}_{2}\left(p_{2}\right) \\
& \times\left[\left(m_{1}-m_{2}\right) f_{1}\left(q^{2}\right)+\left(m_{1}+m_{2}\right)\right. \\
& \left.\times g_{1}\left(q^{2}\right) \gamma_{5}\right] u_{1}\left(p_{1}\right),
\end{aligned}
$$

where contributions from the form factors $f_{3}$ and $g_{3}$ can be neglected. ${ }^{3}$ The factorizable contributions to $A$ and $B$ terms finally read

\footnotetext{
${ }^{2}$ Here we follow the PDG convention $\left\langle 0\left|A_{\mu}(0)\right| P(\boldsymbol{q})\right\rangle=i f_{P} q_{\mu}$ for the decay constant. This differs from the sign convention used in [26].

${ }^{3}$ To see the possible corrections from the form factors $f_{3}$ and $g_{3}$ for kaon or $\eta$ production in the final state, we notice that $m_{P}^{2} / m_{\Lambda_{c}}^{2}=0.047$ for the kaon and 0.057 for the $\eta$. Since the form factor $f_{3}$ is much smaller than $f_{1}$ (see, e.g., Table IV of [17]), while $g_{3}$ is of the same order as $g_{1}$, it follows that the form factor $f_{3}$ can be safely neglected in the factorizable amplitude, while $g_{3}$ could make $\sim 5 \%$ corrections for kaon or $\eta$ production. For simplicity, we drop all the contributions from $f_{3}$ and $g_{3}$.
}

$\left.A^{\mathrm{fac}}\right|_{\mathrm{CF}}=\frac{G_{F}}{\sqrt{2}} a_{1,2} V_{u d}^{*} V_{c s} f_{P}\left(m_{\mathcal{B}_{c}}-m_{\mathcal{B}}\right) f_{1}\left(q^{2}\right)$,

$\left.B^{\mathrm{fac}}\right|_{\mathrm{CF}}=-\frac{G_{F}}{\sqrt{2}} a_{1,2} V_{u d}^{*} V_{c s} f_{P}\left(m_{\mathcal{B}_{c}}+m_{\mathcal{B}}\right) g_{1}\left(q^{2}\right)$,

where the choice of $a_{i}$ can be referred to Eq. (10).

Likewise, the $S$ - and $P$-wave amplitudes for SCS processes are given by

$\begin{aligned}\left.A^{\mathrm{fac}}\right|_{\mathrm{SCS}} & =\frac{G_{F}}{\sqrt{2}} a_{1,2} V_{u q}^{*} V_{c q} f_{P}\left(m_{\mathcal{B}_{c}}-m_{\mathcal{B}}\right) f_{1}\left(q^{2}\right), \\ \left.B^{\mathrm{fac}}\right|_{\mathrm{SCS}} & =-\frac{G_{F}}{\sqrt{2}} a_{1,2} V_{u q}^{*} V_{c q} f_{P}\left(m_{\mathcal{B}_{c}}+m_{\mathcal{B}}\right) g_{1}\left(q^{2}\right),\end{aligned}$

where the flavor of the down-type quark $q, d$, or $s$ depends on the process. If $P=\eta_{8}$, both flavors contribute, for example,

$$
\begin{aligned}
A^{\mathrm{fac}}\left(\Lambda_{c}^{+} \rightarrow p \eta\right)= & \frac{G_{F}}{\sqrt{2}} a_{2}\left(V_{c s} V_{u s} f_{\eta}^{s}+\frac{1}{\sqrt{2}} V_{c d} V_{u d} f_{\eta}^{q}\right) \\
& \times\left(m_{\Lambda_{c}}-m_{p}\right) f_{1}^{\Lambda_{c} p}\left(m_{\eta}^{2}\right), \\
B^{\mathrm{fac}}\left(\Lambda_{c}^{+} \rightarrow p \eta\right)= & -\frac{G_{F}}{\sqrt{2}} a_{2}\left(V_{c s} V_{u s} f_{\eta}^{s}+\frac{1}{\sqrt{2}} V_{c d} V_{u d} f_{\eta}^{q}\right) \\
& \times\left(m_{\Lambda_{c}}+m_{p}\right) g_{1}^{\Lambda_{c} p}\left(m_{\eta}^{2}\right),
\end{aligned}
$$

where the decay constants are defined by

$$
\begin{aligned}
\left\langle\eta\left|\bar{q} \gamma_{\mu}\left(1-\gamma_{5}\right) q\right| 0\right\rangle & =i \frac{1}{\sqrt{2}} f_{\eta}^{q} q_{\mu}, \\
\left\langle\eta\left|\bar{s} \gamma_{\mu}\left(1-\gamma_{5}\right) s\right| 0\right\rangle & =i f_{\eta}^{s} q_{\mu} .
\end{aligned}
$$

We follow [32] to use $f_{\eta}^{q}=107 \mathrm{MeV}$ and $f_{\eta}^{s}=-112 \mathrm{MeV}$. Notice that in the case of $\pi^{0}$ production in the final state, one should replace $a_{2}$ by $-a_{2} / \sqrt{2}$ in the factorizable amplitude, where the extra factor of $-1 / \sqrt{2}$ comes from the wave function of the $\pi^{0}, \pi^{0}=(u \bar{u}-d \bar{d}) / \sqrt{2}$.

\section{The parametrization of form factors}

There are two different nonperturbative parameters in factorizable amplitudes, the decay constant and the form factor (FF). There exist some efforts for estimating the FFs for $\Xi_{c} \rightarrow \mathcal{B}$ transition [17,33-35]. In this work we prefer to work out FFs for $\Xi_{c}-\mathcal{B}$ transition and baryonic matrix elements all within the MIT bag model [36]. Since the decay rates and decay asymmetries are sensitive to the relative sign between factorizable and nonfactorizable amplitudes, it is also desired to have an estimation of FFs in a globally consistent convention.

\footnotetext{
${ }^{4}$ See chapter 18 of [37] for a nice introduction to the MIT bag model. For the evaluation of baryon matrix elements and form factors in this model, see, e.g., [23,34].
} 
TABLE II. Form factors of $\Xi_{c}^{0,+} \rightarrow \mathcal{B P}$ evaluated in the MIT bag model. The calculated results at $q^{2}=q_{\text {max }}^{2}$ are presented in the third/ sixth column. With different involved quark content shown in the second column, the evolution coefficients are shown in the fourth/ seventh column. The physical FFs $f_{1}\left(m_{P}^{2}\right)$ are shown in the fifth column; likewise for $g_{1}\left(m_{P}^{2}\right)$.

\begin{tabular}{|c|c|c|c|c|c|c|c|}
\hline Modes & $(c \bar{q})$ & $f_{1}\left(q_{\max }^{2}\right)$ & $f_{1}\left(m_{P}^{2}\right) / f_{1}\left(q_{\max }^{2}\right)$ & $f_{1}\left(m_{P}^{2}\right)$ & $g_{1}\left(q_{\max }^{2}\right)$ & $g_{1}\left(m_{P}^{2}\right) / g_{1}\left(q_{\max }^{2}\right)$ & $g_{1}\left(m_{P}^{2}\right)$ \\
\hline$\Xi_{c}^{+} \rightarrow \Sigma^{+} \bar{K}^{0}$ & $(c \bar{s})$ & $-\frac{\sqrt{6}}{2} Y_{1}$ & 0.44907 & -0.485 & $-\frac{v}{2}$ & 0.60286 & -0.567 \\
\hline$\Xi_{c}^{+} \rightarrow \Xi^{0} \pi^{+}$ & $(c \bar{s})$ & $-\frac{\sqrt{6}}{2} Y_{1}^{s}$ & 0.49628 & -0.577 & $-\frac{\sqrt{6}}{2} Y_{2}^{s}$ & 0.63416 & -0.667 \\
\hline$\Xi_{c}^{0} \rightarrow \Lambda \bar{K}^{0}$ & $(c \bar{s})$ & $\frac{1}{2} Y_{1}$ & 0.38700 & 0.171 & $\frac{1}{2} Y_{2}$ & 0.55337 & 0.213 \\
\hline$\Xi_{c}^{0} \rightarrow \Sigma^{0} \bar{K}^{0}$ & $(c \bar{s})$ & & 0.44929 & 0.343 & & 0.60304 & 0.401 \\
\hline$\Xi_{c}^{0} \rightarrow \Xi^{-} \pi^{+}$ & $(c \bar{s})$ & $-\frac{\sqrt{6}}{2} Y_{1}^{s}$ & 0.49911 & -0.581 & & 0.63636 & -0.669 \\
\hline$\Xi_{c}^{+} \rightarrow \Sigma^{0} \pi^{+}$ & $(c \bar{d})$ & & 0.36045 & 0.275 & & 0.52523 & 0.350 \\
\hline$\Xi_{c}^{+} \rightarrow \Lambda \pi^{+}$ & $(c \bar{d})$ & $-\frac{1}{2} Y_{1}$ & 0.30260 & -0.134 & $-\frac{1}{2} Y_{2}$ & 0.47622 & -0.183 \\
\hline$\Xi_{c}^{+} \rightarrow \Sigma^{+} \pi^{0}$ & $(c \bar{d})$ & $-\frac{\sqrt{6}}{2} Y_{1}$ & 0.35774 & -0.387 & & 0.52294 & -0.492 \\
\hline$\Xi_{c}^{+} \rightarrow \Sigma^{+} \eta$ & $(c \bar{d})$ & $-\frac{\sqrt{6}}{2} Y_{1}$ & 0.41371 & -0.447 & $-\frac{\sqrt{2}}{2}$ & 0.57735 & -0.543 \\
\hline$\Xi_{c}^{+} \rightarrow \Xi^{0} K^{+}$ & $(c \bar{s})$ & $-\frac{\sqrt{6}}{2} Y_{1}^{s}$ & 0.55058 & -0.641 & $-\frac{\sqrt{6}}{2} Y_{2}^{s}$ & 0.68080 & -0.716 \\
\hline$\Xi_{c}^{0} \rightarrow \Lambda \eta$ & $(c \bar{d})$ & $\frac{1}{2} Y_{1}$ & 0.34715 & 0.153 & $\frac{1}{2} Y_{2}$ & 0.52343 & 0.201 \\
\hline$\Xi_{c}^{0} \rightarrow \Sigma^{0} \eta$ & $(c \bar{d})$ & $\frac{\sqrt{3}}{2} Y_{1}$ & 0.41395 & 0.316 & $\frac{\sqrt{3}}{2} Y_{2}$ & 0.57754 & 0.384 \\
\hline$\Xi_{c}^{0} \rightarrow \Lambda \pi^{0}$ & $(c \bar{d})$ & $\frac{1}{2} Y_{1}$ & 0.30019 & 0.132 & $\frac{1}{2} Y_{2}$ & 0.47410 & 0.182 \\
\hline$\Xi_{c}^{0} \rightarrow \Sigma^{0} \pi^{0}$ & $(c \bar{d})$ & $\frac{\sqrt{3}}{2} Y_{1}$ & 0.35795 & 0.274 & $\frac{\sqrt{3}}{2} Y_{2}$ & 0.52311 & 0.348 \\
\hline$\Xi_{c}^{0} \rightarrow \Sigma^{-} \pi^{+}$ & $(c \bar{d})$ & $\frac{\sqrt{6}}{2} Y_{1}$ & 0.36183 & 0.391 & $\frac{\sqrt{6}}{2} Y_{2}$ & 0.52638 & 0.496 \\
\hline$\Xi_{c}^{0} \rightarrow \Xi^{-} K^{+}$ & $(c \bar{s})$ & $-\frac{\sqrt{6}}{2} Y_{1}^{s}$ & 0.55371 & -0.644 & $-\frac{\sqrt{6}}{2} Y_{2}^{s}$ & 0.68316 & -0.719 \\
\hline
\end{tabular}
FF as

In this work we follow [38] to write the $q^{2}$ dependence of

$f_{i}\left(q^{2}\right)=\frac{f_{i}(0)}{\left(1-q^{2} / m_{V}^{2}\right)^{2}}, \quad g_{i}\left(q^{2}\right)=\frac{g_{i}(0)}{\left(1-q^{2} / m_{A}^{2}\right)^{2}}$,

where $m_{V}=2.01 \mathrm{GeV}, m_{A}=2.42 \mathrm{GeV}$ for the $(c \bar{d})$ quark content, and $m_{V}=2.11 \mathrm{GeV}, m_{A}=2.54 \mathrm{GeV}$ for the $(c \bar{s})$ quark content. In the zero recoil limit where $q_{\max }^{2}=\left(m_{i}-m_{f}\right)^{2}$, FFs can be expressed within the MIT bag model as [23]

$$
\begin{aligned}
f_{1}^{\mathcal{B}_{f} \mathcal{B}_{i}}\left(q_{\text {max }}^{2}\right)= & \left\langle\mathcal{B}_{f}^{\uparrow}\left|b_{q_{1}}^{\dagger} b_{q_{2}}\right| \mathcal{B}_{i}^{\uparrow}\right\rangle \\
& \times \int d^{3} \boldsymbol{r}\left(u_{q_{1}}(r) u_{q_{2}}(r)+v_{q_{1}}(r) v_{q_{2}}(r)\right), \\
g_{1}^{\mathcal{B}_{f} \mathcal{B}_{i}}\left(q_{\text {max }}^{2}\right)= & \left\langle\mathcal{B}_{f}^{\uparrow}\left|b_{q_{1}}^{\dagger} b_{q_{2}} \sigma_{z}\right| \mathcal{B}_{i}^{\uparrow}\right\rangle \\
& \times \int d^{3} \boldsymbol{r}\left(u_{q_{1}}(r) u_{q_{2}}(r)-\frac{1}{3} v_{q_{1}}(r) v_{q_{2}}(r)\right),
\end{aligned}
$$

where $u(r)$ and $v(r)$ are the large and small components, respectively, of the quark wave function in the bag model. FFs at different $q^{2}$ are related via

$$
\begin{aligned}
f_{i}\left(q_{2}^{2}\right) & =\frac{\left(1-q_{1}^{2} / m_{V}^{2}\right)^{2}}{\left(1-q_{2}^{2} / m_{V}^{2}\right)^{2}} f_{i}\left(q_{1}^{2}\right), \\
g_{i}\left(q_{2}^{2}\right) & =\frac{\left(1-q_{1}^{2} / m_{A}^{2}\right)^{2}}{\left(1-q_{2}^{2} / m_{A}^{2}\right)^{2}} g_{i}\left(q_{1}^{2}\right) .
\end{aligned}
$$

This allows us to obtain the physical FF at $q^{2}=m_{P}^{2}$.

It is obvious that the FF at $q_{\max }^{2}$ is determined only by the baryons in initial and final states. However, its evolution with $q^{2}$ is governed by the relevant quark content. Such a dependence is reflected in Table II, in which the quark contents are shown in the second column. In the zero recoil limit, the FFs at $q_{\max }^{2}$ calculated from Eq. (18) are presented in the third and sixth columns. And then in the fourth and seventh columns, the evolution of FFs from $q^{2}=q_{\max }^{2}$ to $q^{2}=m_{P}^{2}$ are derived according to Eq. (20). The bag integrals $Y_{1,2}^{(s)}$ are defined by

$$
\begin{aligned}
& Y_{1}=4 \pi \int r^{2} d r\left(u_{u} u_{c}+v_{u} v_{c}\right), \\
& Y_{1}^{s}=4 \pi \int r^{2} d r\left(u_{s} u_{c}+v_{s} v_{c}\right), \\
& Y_{2}=4 \pi \int r^{2} d r\left(u_{u} u_{c}-\frac{1}{3} v_{u} v_{c}\right), \\
& Y_{2}^{s}=4 \pi \int r^{2} d r\left(u_{s} u_{c}-\frac{1}{3} v_{s} v_{c}\right) .
\end{aligned}
$$


The model parameters are adopted from [26] and references therein. Numerically, we have $Y_{1}=0.88$, $Y_{1}^{s}=0.95, Y_{2}=0.77, Y_{2}^{s}=0.86$, which are consistent with the corresponding numbers in [23].

\section{Nonfactorizable contribution}

We work in the framework of the pole model to estimate nonfactorizable contributions. It is known that the S-wave amplitude is dominated by the low-lying $1 / 2^{-}$resonances, while the $P$-wave one is governed by the ground-state $1 / 2^{+}$ pole. The general formulas for $A$ ( $S$-wave) and $B$ ( $P$-wave) terms in the pole model are given by $[34]^{5}$

$$
\begin{aligned}
A^{\text {pole }} & =-\sum_{\mathcal{B}_{n}^{*}\left(1 / 2^{-}\right)}\left[\frac{g_{\mathcal{B}_{f} \mathcal{B}_{n}^{*} P} b_{n^{*} i}}{m_{i}-m_{n^{*}}}+\frac{b_{f n^{*}} g_{\mathcal{B}_{n}^{*} \mathcal{B}_{i} P}}{m_{f}-m_{n^{*}}}\right], \\
B^{\text {pole }} & =\sum_{\mathcal{B}_{n}}\left[\frac{g_{\mathcal{B}_{f} \mathcal{B}_{n} P} a_{n i}}{m_{i}-m_{n}}+\frac{a_{f n} g_{\mathcal{B}_{n} \mathcal{B}_{i} P}}{m_{f}-m_{n}}\right],
\end{aligned}
$$

where $a_{i j}, b_{i j}$ are the baryon matrix elements defined by

$$
\begin{aligned}
\left\langle\mathcal{B}_{n}|\mathcal{H}| \mathcal{B}_{i}\right\rangle & =\bar{u}_{n}\left(a_{n i}-b_{n i} \gamma_{5}\right) u_{i}, \\
\left\langle\mathcal{B}_{i}^{*}\left(1 / 2^{-}\right)|\mathcal{H}| \mathcal{B}_{j}\right\rangle & =\bar{u}_{i^{*}} b_{i^{*} j} u_{j} .
\end{aligned}
$$

In the soft-meson limit, the intermediate excited $1 / 2^{-}$states in the $S$ - wave amplitude can be summed up and reduced to a commutator term [26],

$$
A^{\mathrm{com}}=-\frac{\sqrt{2}}{f_{P^{a}}}\left\langle\mathcal{B}_{f}\left|\left[Q_{5}^{a}, H_{\mathrm{eff}}^{\mathrm{PV}}\right]\right| \mathcal{B}_{i}\right\rangle=\frac{\sqrt{2}}{f_{P^{a}}}\left\langle\mathcal{B}_{f}\left|\left[Q^{a}, H_{\mathrm{eff}}^{\mathrm{PC}}\right]\right| \mathcal{B}_{i}\right\rangle
$$

with

$$
Q^{a}=\int d^{3} x \bar{q} \gamma^{0} \frac{\lambda^{a}}{2} q, \quad Q_{5}^{a}=\int d^{3} x \bar{q} \gamma^{0} \gamma_{5} \frac{\lambda^{a}}{2} q .
$$

By applying the generalized Goldberger-Treiman relation

$$
g_{\mathcal{B}^{\prime} \mathcal{B} P^{a}}=\frac{\sqrt{2}}{f_{P^{a}}}\left(m_{\mathcal{B}}+m_{\mathcal{B}^{\prime}}\right) g_{\mathcal{B}^{\prime} \mathcal{B}}^{A},
$$

the $P$-wave amplitude can be simplified to

$$
B^{\text {pole }}=\frac{\sqrt{2}}{f_{P^{a}}} \sum_{\mathcal{B}_{n}}\left[g_{\mathcal{B}_{f} \mathcal{B}_{n}}^{A} \frac{m_{f}+m_{n}}{m_{i}-m_{n}} a_{n i}+a_{f n} \frac{m_{i}+m_{n}}{m_{f}-m_{n}} g_{\mathcal{B}_{n} \mathcal{B}_{i}}^{A}\right] .
$$

Therefore, the two master equations Eqs. (24) and (27) for the nonfactorizable contributions in the pole model rely on

\footnotetext{
${ }^{5}$ Note that we have corrected the sign of the $B$ term in [26].

${ }^{6}$ The applied relation $\left[Q_{5}, H_{\mathrm{eff}}^{\mathrm{pv}}\right]=-\left[Q, H_{\mathrm{eff}}^{\mathrm{pc}}\right]$ differs from that in [26] in sign.
}

the commutator terms and the axial-vector form factor $g_{\mathcal{B}^{\prime} \mathcal{B}}^{A}$, which is calculated in the MIT bag model in this work.

\section{S-wave amplitude}

We have deduced that the $S$-wave amplitude is determined by the commutator terms of conserving charge $Q^{a}$ and the parity-conserving part of the Hamiltonian. In the following we list the expressions of $A^{\text {com }}$ according to Eq. (24),

$$
\begin{aligned}
A^{\mathrm{com}}\left(\mathcal{B}_{i} \rightarrow \mathcal{B}_{f} \pi^{ \pm}\right) & =\frac{1}{f_{\pi}}\left\langle\mathcal{B}_{f}\left|\left[I_{\mp}, H_{\mathrm{eff}}^{\mathrm{PC}}\right]\right| \mathcal{B}_{i}\right\rangle, \\
A^{\mathrm{com}}\left(\mathcal{B}_{i} \rightarrow \mathcal{B}_{f} \pi^{0}\right) & =\frac{\sqrt{2}}{f_{\pi}}\left\langle\mathcal{B}_{f}\left|\left[I_{3}, H_{\mathrm{eff}}^{\mathrm{PC}}\right]\right| \mathcal{B}_{i}\right\rangle, \\
A^{\mathrm{com}}\left(\mathcal{B}_{i} \rightarrow \mathcal{B}_{f} \eta_{8}\right) & =\sqrt{\frac{3}{2}} \frac{1}{f_{\eta_{8}}}\left\langle\mathcal{B}_{f}\left|\left[Y, H_{\mathrm{eff}}^{\mathrm{PC}}\right]\right| \mathcal{B}_{i}\right\rangle, \\
A^{\mathrm{com}}\left(\mathcal{B}_{i} \rightarrow \mathcal{B}_{f} K^{ \pm}\right) & =\frac{1}{f_{K}}\left\langle\mathcal{B}_{f}\left|\left[V_{\mp}, H_{\mathrm{eff}}^{\mathrm{PC}}\right]\right| \mathcal{B}_{i}\right\rangle, \\
A^{\operatorname{com}}\left(\mathcal{B}_{i} \rightarrow \mathcal{B}_{f} \bar{K}^{0}\right) & =\frac{1}{f_{K}}\left\langle\mathcal{B}_{f}\left|\left[U_{+}, H_{\mathrm{eff}}^{\mathrm{PC}}\right]\right| \mathcal{B}_{i}\right\rangle, \\
A^{\operatorname{com}}\left(\mathcal{B}_{i} \rightarrow \mathcal{B}_{f} K^{0}\right) & =\frac{1}{f_{K}}\left\langle\mathcal{B}_{f}\left|\left[U_{-}, H_{\mathrm{eff}}^{\mathrm{PC}}\right]\right| \mathcal{B}_{i}\right\rangle,
\end{aligned}
$$

where we have introduced the isospin, $U$-spin, and $V$-spin ladder operators with

$$
\begin{aligned}
& I_{+}|d\rangle=|u\rangle, \quad I_{-}|u\rangle=|d\rangle, \quad U_{+}|s\rangle=|d\rangle, \\
& U_{-}|d\rangle=|s\rangle, \quad V_{+}|s\rangle=|u\rangle, \quad V_{-}|u\rangle=|s\rangle .
\end{aligned}
$$

In Eq. (28), $\eta_{8}$ is the octet component of the $\eta$ and $\eta^{\prime}$

$\eta=\cos \theta \eta_{8}-\sin \theta \eta_{0}, \quad \eta^{\prime}=\sin \theta \eta_{8}+\cos \theta \eta_{0}$,

with $\theta=-15.4^{\circ}$ [32]. For the decay constant $f_{\eta_{8}}$, we follow [32] to use $f_{\eta_{8}}=f_{8} \cos \theta$ with $f_{8}=1.26 f_{\pi}$. Hypercharge $Y$, the conserving charge for processes involving $\eta_{8}$ in the final state, is taken to be $Y=$ $B+S-C$ as shown in [26]. The baryon matrix elements of commutators in Eq. (28), after the action of the ladder operators on baryon wave functions shown in Appendix $\mathrm{B}$, can be further reduced to pure matrix elements of effective Hamiltonian, denoted by $a_{\mathcal{B}^{\prime} \mathcal{B}} \equiv$ $\left\langle\mathcal{B}^{\prime}\left|H_{\text {eff }}^{\mathrm{PC}}\right| \mathcal{B}\right\rangle$. Then in terms of $a_{\mathcal{B}^{\prime} \mathcal{B}}$, nonfactorizable contributions to $S$-wave amplitudes for charmed baryon decays are calculable. 
For the Cabibbo-favored processes, we have

$$
\begin{aligned}
A^{\mathrm{com}}\left(\Lambda_{c}^{+} \rightarrow p \bar{K}^{0}\right) & =-\frac{1}{f_{K}} a_{\Sigma^{+} \Lambda_{c}^{+}}, & A^{\mathrm{com}}\left(\Lambda_{c}^{+} \rightarrow \Lambda \pi^{+}\right) & =0, \\
A^{\mathrm{com}}\left(\Lambda_{c}^{+} \rightarrow \Sigma^{0} \pi^{+}\right) & =-\frac{\sqrt{2}}{f_{\pi}} a_{\Sigma^{+} \Lambda_{c}^{+}}, & A^{\mathrm{com}}\left(\Lambda_{c}^{+} \rightarrow \Sigma^{+} \pi^{0}\right) & =\frac{\sqrt{2}}{f_{\pi}} a_{\Sigma^{+} \Lambda_{c}^{+}}, \\
A^{\mathrm{com}}\left(\Lambda_{c}^{+} \rightarrow \Xi^{0} K^{+}\right) & =\frac{1}{f_{K}} a_{\Sigma^{+} \Lambda_{c}^{+}}, & A^{\mathrm{com}}\left(\Lambda_{c}^{+} \rightarrow \Sigma^{+} \eta_{8}\right) & =\frac{\sqrt{2}}{\sqrt{3} f_{\eta_{8}}} a_{\Sigma_{c}^{+} \Sigma^{+}},
\end{aligned}
$$

and

$$
\begin{aligned}
A^{\mathrm{com}}\left(\Xi_{c}^{+} \rightarrow \Sigma^{+} \bar{K}^{0}\right) & =\frac{1}{f_{K}} a_{\Sigma^{+} \Lambda_{c}^{+}}, & A^{\mathrm{com}}\left(\Xi_{c}^{+} \rightarrow \Xi^{0} \pi^{+}\right) & =-\frac{1}{f_{\pi}} a_{\Xi^{0} \Xi_{c}^{0}}, \\
A^{\mathrm{com}}\left(\Xi_{c}^{0} \rightarrow \Lambda \bar{K}^{0}\right) & =\frac{1}{f_{K}} \frac{\sqrt{6}}{2} a_{\Xi^{0} \Xi_{c}^{0},}, & A^{\mathrm{com}}\left(\Xi_{c}^{0} \rightarrow \Sigma^{0} \bar{K}^{0}\right) & =-\frac{1}{f_{K}} \frac{\sqrt{2}}{2} a_{\Xi^{0} \Xi_{c}^{0},} \\
A^{\mathrm{com}}\left(\Xi_{c}^{0} \rightarrow \Sigma^{+} K^{-}\right) & =\frac{1}{f_{K}} a_{\Xi^{0} \Xi_{c}^{0}}, & A^{\mathrm{com}}\left(\Xi_{c}^{0} \rightarrow \Xi^{0} \pi^{0}\right) & =\frac{\sqrt{2}}{f_{\pi}} a_{\Xi^{0} \Xi_{c}^{0}}, \\
A^{\mathrm{com}}\left(\Xi_{c}^{0}\right. & \left.\rightarrow \Xi^{0} \eta_{8}\right)=\frac{\sqrt{6}}{f_{\eta_{8}}} a_{\Xi^{0} \Xi_{c}^{0}}, & A^{\mathrm{com}}\left(\Xi_{c}^{0} \rightarrow \Xi^{-} \pi^{+}\right) & =\frac{1}{f_{\pi}} a_{\Xi^{0} \Xi_{c}^{0}} .
\end{aligned}
$$

For singly Cabibbo-suppressed processes we have

$$
\begin{array}{rlrl}
A^{\mathrm{com}}\left(\Xi_{c}^{+} \rightarrow \Sigma^{0} \pi^{+}\right) & =-\frac{1}{f_{\pi}}\left(\sqrt{2} a_{\Sigma^{+} \Xi_{c}^{+}}+a_{\Sigma^{0} \Xi_{c}^{0}}\right), & A^{\mathrm{com}}\left(\Xi_{c}^{+} \rightarrow \Sigma^{+} \pi^{0}\right)=\frac{1}{\sqrt{2} f_{\pi}} a_{\Sigma^{+} \Xi_{c}^{+}}, \\
A^{\mathrm{com}}\left(\Xi_{c}^{+} \rightarrow p \bar{K}^{0}\right)=-\frac{1}{f_{K}}\left(a_{\Sigma^{+} \Xi_{c}^{+}}-a_{p \Lambda_{c}^{+}}\right), & A^{\mathrm{com}}\left(\Xi_{c}^{+} \rightarrow \Lambda \pi^{+}\right)=-\frac{1}{f_{\pi}} a_{\Lambda \Xi_{c}^{0}}, \\
A^{\mathrm{com}}\left(\Xi_{c}^{+} \rightarrow \Sigma^{+} \eta_{8}\right)=\frac{\sqrt{6}}{2} \frac{1}{f_{\eta_{8}}} a_{\Sigma^{+} \Xi_{c}^{+}}, & A^{\mathrm{com}}\left(\Xi_{c}^{+} \rightarrow \Xi^{0} K^{+}\right)=\frac{1}{f_{K}} a_{\Sigma^{+} \Xi_{c}^{+}},
\end{array}
$$

and

$$
\begin{array}{rlrl}
A^{\mathrm{com}}\left(\Xi_{c}^{0} \rightarrow \Xi^{0} K^{0}\right) & =\frac{1}{f_{K}}\left(-\frac{\sqrt{2}}{2} a_{\Sigma^{0} \Xi_{c}^{0}}+\frac{\sqrt{6}}{2} a_{\Lambda \Xi_{c}^{0}}\right), & A^{\mathrm{com}}\left(\Xi_{c}^{0} \rightarrow \Lambda \pi^{0}\right) & =\frac{1}{\sqrt{2} f_{\pi}} a_{\Lambda \Xi_{c}^{0}}, \\
A^{\mathrm{com}}\left(\Xi_{c}^{0} \rightarrow \Xi^{-} K^{+}\right) & =-\frac{1}{f_{K}}\left(\frac{\sqrt{2}}{2} a_{\Sigma^{0} \Xi_{c}^{0}}+\frac{\sqrt{6}}{2} a_{\Lambda \Xi_{c}^{0}}\right), & A^{\mathrm{com}}\left(\Xi_{c}^{0} \rightarrow \Sigma^{-} \pi^{+}\right)=\frac{\sqrt{2}}{f_{\pi}} a_{\Sigma^{0} \Xi_{c}^{0}}, \\
A^{\mathrm{com}}\left(\Xi_{c}^{0} \rightarrow p K^{-}\right)=-\frac{1}{f_{K}}\left(\frac{\sqrt{2}}{2} a_{\Sigma^{0} \Xi_{c}^{0}}+\frac{\sqrt{6}}{2} a_{\Lambda \Xi_{c}^{0}}\right), & A^{\mathrm{com}}\left(\Xi_{c}^{0} \rightarrow \Sigma^{0} \pi^{0}\right)=\frac{1}{\sqrt{2} f_{\pi}} a_{\Sigma^{0} \Xi_{c}^{0}}, \\
A^{\mathrm{com}}\left(\Xi_{c}^{0} \rightarrow n \bar{K}^{0}\right)=\frac{1}{f_{K}}\left(\frac{\sqrt{2}}{2} a_{\Sigma^{0} \Xi_{c}^{0}}-\frac{\sqrt{6}}{2} a_{\Lambda \Xi_{c}^{0}}\right), & A^{\mathrm{com}}\left(\Xi_{c}^{0} \rightarrow \Sigma^{+} \pi^{-}\right)=-\frac{\sqrt{2}}{f_{\pi}} a_{\Sigma^{0} \Xi_{c}^{0} .} . \\
A^{\operatorname{com}}\left(\Xi_{c}^{0} \rightarrow \Lambda \eta_{8}\right)=\frac{\sqrt{6}}{2} \frac{1}{f_{\eta_{8}}} a_{\Lambda \Xi_{c}^{0}}, & A^{\operatorname{com}}\left(\Xi_{c}^{0} \rightarrow \Sigma^{0} \eta_{8}\right)=\frac{\sqrt{6}}{2} \frac{1}{f_{\eta_{8}}} a_{\Sigma^{0} \Xi_{c}^{0}} .
\end{array}
$$

The nonfactorizable $S$-wave amplitudes for SCS decays of $\Lambda_{c}^{+}$can be found in [26]. The evaluation of the baryon matrix elements $a_{\mathcal{B}^{\prime} \mathcal{B}}$ in the MIT bag model and results are presented in Appendix D 1.

\section{P-wave amplitude}

Through the generalized Goldberger-Treiman relation Eq. (26), the strong coupling of $\mathcal{B}^{\prime} \mathcal{B} M$ can be expressed in terms of the axial-vector form factor $g_{\mathcal{B}^{\prime} \mathcal{B}}^{A}$. Based on Eq. (27), $P$-wave amplitudes are given as follows. For Cabibbo-favored processes we have 


$$
\begin{aligned}
& B^{\mathrm{ca}}\left(\Lambda_{c}^{+} \rightarrow p \bar{K}^{0}\right)=\frac{1}{f_{K}}\left(g_{p \Sigma^{+}}^{\left(\bar{K}^{0}\right)} \frac{m_{p}+m_{\Sigma^{+}}}{m_{\Lambda_{c}^{+}}-m_{\Sigma^{+}}} a_{\Sigma^{+} \Lambda_{c}^{+}}\right) \\
& B^{\mathrm{ca}}\left(\Lambda_{c}^{+} \rightarrow \Lambda \pi^{+}\right)=\frac{1}{f_{\pi}}\left(a_{\Lambda \Sigma_{c}^{0}} \frac{m_{\Lambda_{c}^{+}}+m_{\Sigma_{c}^{0}}}{m_{\Lambda}-m_{\Sigma_{c}^{0}}} g_{\Sigma_{c}^{0} \Lambda_{c}^{+}}^{A\left(\pi^{+}\right)}+g_{\Lambda \Sigma^{+}}^{A\left(\pi^{+}\right)} \frac{m_{\Lambda}+m_{\Sigma^{+}}}{m_{\Lambda_{c}^{+}}-m_{\Sigma^{+}}} a_{\Sigma^{+} \Lambda_{c}^{+}}\right), \\
& B^{\mathrm{ca}}\left(\Lambda_{c}^{+} \rightarrow \Sigma^{0} \pi^{+}\right)=\frac{1}{f_{\pi}}\left(a_{\Sigma^{0} \Sigma_{c}^{0}} \frac{m_{\Lambda_{c}^{+}}+m_{\Sigma_{c}^{0}}}{m_{\Sigma^{0}}-m_{\Sigma_{c}^{0}}} g_{\Sigma_{c}^{0} \Lambda_{c}^{+}}^{A\left(\pi^{+}\right)}+g_{\Sigma^{0} \Sigma^{+}}^{A\left(\pi^{+}\right)} \frac{m_{\Sigma^{0}}+m_{\Sigma^{+}}}{m_{\Lambda_{c}^{+}}-m_{\Sigma^{+}}} a_{\Sigma^{+}} \Lambda_{c}^{+}\right), \\
& B^{\mathrm{ca}}\left(\Lambda_{c}^{+} \rightarrow \Sigma^{+} \pi^{0}\right)=\frac{\sqrt{2}}{f_{\pi}}\left(a_{\Sigma^{+} \Lambda_{c}^{+}} \frac{m_{\Lambda_{c}^{+}}+m_{\Lambda_{c}^{+}}}{m_{\Sigma^{+}}-m_{\Lambda_{c}^{+}}} g_{\Lambda_{c}^{+} \Lambda_{c}^{+}}^{A\left(\pi^{0}\right)}+a_{\Sigma^{+} \Sigma_{c}^{+}} \frac{m_{\Lambda_{c}^{+}}+m_{\Sigma_{c}^{+}}}{m_{\Sigma^{+}}-m_{\Sigma_{c}^{+}}} g_{\Sigma_{c}^{+} \Lambda_{c}^{+}}^{A\left(\pi^{0}\right)}+g_{\Sigma^{+} \Sigma^{+}}^{A\left(\pi^{0}\right)} \frac{m_{\Sigma^{+}}+m_{\Sigma^{+}}}{m_{\Lambda_{c}^{+}}-m_{\Sigma^{+}}} a_{\Sigma^{+} \Lambda_{c}^{+}}\right), \\
& B^{\mathrm{ca}}\left(\Lambda_{c}^{+} \rightarrow \Xi^{0} K^{+}\right)=\frac{1}{f_{K}}\left(g_{\Xi^{0} \Sigma^{+}}^{A\left(K^{+}\right)} \frac{m_{\Xi^{0}}+m_{\Sigma^{+}}}{m_{\Lambda_{c}^{+}}-m_{\Sigma^{+}}} a_{\Sigma^{+} \Lambda_{c}^{+}}\right),
\end{aligned}
$$

and

$$
\begin{aligned}
& B^{\mathrm{ca}}\left(\Xi_{c}^{+} \rightarrow \Sigma^{+} \bar{K}^{0}\right)=\frac{1}{f_{K}}\left(a_{\Sigma^{+} \Lambda_{c}^{+}} \frac{m_{\Xi_{c}^{+}}+m_{\Lambda_{c}^{+}}}{m_{\Sigma^{+}}-m_{\Lambda_{c}^{+}}} g_{\Lambda_{c}^{+} \Xi_{c}^{+}}^{A\left(\bar{K}^{0}\right)}+a_{\Sigma^{+} \Sigma_{c}^{+}} \frac{m_{\Xi_{c}^{+}}+m_{\Sigma_{c}^{+}}}{m_{\Sigma^{+}}-m_{\Sigma_{c}^{+}}} g_{\Sigma_{c}^{+} \Xi_{c}^{+}}^{A\left(\bar{K}^{0}\right)}\right), \\
& B^{\mathrm{ca}}\left(\Xi_{c}^{+} \rightarrow \Xi^{0} \pi^{+}\right)=\frac{1}{f_{\pi}}\left(a_{\Xi^{0} \Xi_{c}^{0}} \frac{m_{\Xi_{c}^{+}}+m_{\Xi_{c}^{0}}}{m_{\Xi^{0}}-m_{\Xi_{c}^{0}}} g_{\Xi_{c}^{0} \Xi_{c}^{+}}^{A\left(\pi^{+}\right)}+a_{\Xi^{0} \Xi_{c}^{\prime 0}} \frac{m_{\Xi_{c}^{+}}+m_{\Xi_{c}^{\prime 0}}}{m_{\Xi^{0}}-m_{\Xi_{c}^{\prime 0}}^{A\left(\pi^{+}\right)}} g_{\Xi_{c}^{\prime 0} \Xi_{c}^{+}}\right), \\
& B^{\mathrm{ca}}\left(\Xi_{c}^{0} \rightarrow \Lambda \bar{K}^{0}\right)=\frac{1}{f_{K}}\left(a_{\Lambda \Sigma_{c}^{0}} \frac{m_{\Xi_{c}^{0}}+m_{\Sigma_{c}^{0}}}{m_{\Lambda}-m_{\Sigma_{c}^{0}}} g_{\Sigma_{c}^{0} \Xi_{c}^{0}}^{A\left(\bar{K}^{0}\right)}+g_{\Lambda \Xi^{0}}^{A\left(\bar{K}^{0}\right)} \frac{m_{\Lambda}+m_{\Xi^{0}}}{m_{\Xi_{c}^{0}}-m_{\Xi^{0}}} a_{\Xi^{0} \Xi_{c}^{0}}\right), \\
& B^{\mathrm{ca}}\left(\Xi_{c}^{0} \rightarrow \Sigma^{0} \bar{K}^{0}\right)=\frac{1}{f_{K}}\left(a_{\Sigma^{0} \Sigma_{c}^{0}} \frac{m_{\Xi_{c}^{0}}+m_{\Sigma_{c}^{0}}}{m_{\Sigma^{0}}-m_{\Sigma_{c}^{0}}} g_{\Sigma_{c}^{0} \Xi_{c}^{0}}^{A\left(\bar{K}^{0}\right)}+g_{\Sigma^{0} \Xi^{0}}^{A\left(\bar{K}^{0}\right.} \frac{m_{\Sigma^{0}}+m_{\Xi^{0}}}{m_{\Xi_{c}^{0}}-m_{\Xi^{0}}} a_{\Xi^{0} \Xi_{c}^{0}}\right), \\
& B^{\mathrm{ca}}\left(\Xi_{c}^{0} \rightarrow \Sigma^{+} K^{-}\right)=\frac{1}{f_{K}}\left(g_{\Sigma^{+} \Xi^{0}}^{A\left(K^{-}\right)} \frac{m_{\Sigma^{+}}+m_{\Xi^{0}}}{m_{\Xi_{c}^{0}}-m_{\Xi^{0}}} a_{\Xi^{0} \Xi_{c}^{0}}\right) \\
& B^{\mathrm{ca}}\left(\Xi_{c}^{0} \rightarrow \Xi^{0} \pi^{0}\right)=\frac{\sqrt{2}}{f_{\pi}}\left(a_{\Xi^{0} \Xi_{c}^{0}} \frac{m_{\Xi_{c}^{0}}+m_{\Xi_{c}^{0}}}{m_{\Xi^{0}}-m_{\Xi_{c}^{0}}^{A\left(\pi^{0}\right)}} g_{\Xi_{c}^{0} \Xi_{c}^{0}}+a_{\Xi^{0} \Xi_{c}^{\prime 0}} \frac{m_{\Xi_{c}^{0}}+m_{\Xi_{c}^{\prime 0}}}{m_{\Xi^{0}}-m_{\Xi_{c}^{\prime 0}}} g_{\Xi_{c}^{\prime 0} \Xi_{c}^{0}}^{A\left(\pi^{0}\right)}+g_{\Xi^{0} \Xi^{0}}^{A\left(\pi^{0}\right)} \frac{m_{\Xi^{0}}+m_{\Xi^{0}}}{m_{\Xi_{c}^{0}}-m_{\Xi^{0}}} a_{\Xi^{0} \Xi_{c}^{0}}\right), \\
& B^{\mathrm{ca}}\left(\Xi_{c}^{0} \rightarrow \Xi^{0} \eta_{8}\right)=\frac{\sqrt{2}}{f_{\eta_{8}}}\left(a_{\Xi^{0} \Xi_{c}^{0}} \frac{m_{\Xi_{c}^{0}}+m_{\Xi_{c}^{0}}}{m_{\Xi^{0}}-m_{\Xi_{c}^{0}}} g_{\Xi_{c}^{0} \Xi_{c}^{0}}^{A\left(\eta_{8}\right)}+a_{\Xi^{0} \Xi_{c}^{\prime 0}} \frac{m_{\Xi_{c}^{0}}+m_{\Xi_{c}^{\prime 0}}}{m_{\Xi^{0}}-m_{\Xi_{c}^{\prime 0}}} g_{\Xi_{c}^{\prime 0} \Xi_{c}^{0}}^{A\left(\eta_{8}\right)}+g_{\Xi^{0} \Xi^{0}}^{A\left(\eta_{8}\right)} \frac{m_{\Xi^{0}}+m_{\Xi^{0}}}{m_{\Xi_{c}^{0}}-m_{\Xi^{0}}} a_{\Xi^{0} \Xi_{c}^{0}}\right), \\
& B^{\mathrm{ca}}\left(\Xi_{c}^{0} \rightarrow \Xi^{-} \pi^{+}\right)=\frac{1}{f_{\pi}}\left(g_{\Xi^{-} \Xi^{0}}^{A\left(\pi^{+}\right)} \frac{m_{\Xi^{-}}+m_{\Xi^{0}}}{m_{\Xi_{c}^{0}}-m_{\Xi^{0}}} a_{\Xi^{0} \Xi_{c}^{0}}\right) \text {. }
\end{aligned}
$$

The $P$-wave amplitudes for singly Cabibbo-suppressed processes read

$$
\begin{aligned}
& B^{\mathrm{ca}}\left(\Xi_{c}^{+} \rightarrow \Lambda \pi^{+}\right)=\frac{1}{f_{\pi}}\left(g_{\Lambda \Sigma^{+}}^{A\left(\pi^{+}\right)} \frac{m_{\Lambda}+m_{\Sigma^{+}}}{m_{\Xi_{c}^{+}}-m_{\Sigma^{+}}} a_{\Sigma^{+} \Xi_{c}^{+}}+a_{\Lambda \Xi_{c}^{0}} \frac{m_{\Xi_{c}^{+}}+m_{\Xi_{c}^{0}}}{m_{\Lambda}-m_{\Xi_{c}^{0}}^{A\left(\pi^{+}\right)}} g_{\Xi_{c}^{0} \Xi_{c}^{+}}^{a^{+}}+a_{\Lambda \Xi_{c}^{\prime 0}} \frac{m_{\Xi_{c}^{+}}+m_{\Xi_{c}^{\prime 0}}}{m_{\Lambda}-m_{\Xi_{c}^{\prime 0}}^{A\left(\pi^{+}\right)}} g_{\Xi_{c}^{\prime \prime} \Xi_{c}^{+}}\right), \\
& B^{\mathrm{ca}}\left(\Xi_{c}^{+} \rightarrow \Sigma^{0} \pi^{+}\right)=\frac{1}{f_{\pi}}\left(g_{\Sigma^{0} \Sigma^{+}}^{A\left(\pi^{+}\right)} \frac{m_{\Sigma^{0}}+m_{\Sigma^{+}}}{m_{\Xi_{c}^{+}}-m_{\Sigma^{+}}} a_{\Sigma^{+} \Xi_{c}^{+}}+a_{\Sigma^{0} \Xi_{c}^{0}} \frac{m_{\Xi_{c}^{+}}+m_{\Xi_{c}^{0}}}{m_{\Sigma^{0}}-m_{\Xi_{c}^{0}}^{A\left(\pi^{+}\right)}} g_{\Xi_{c}^{0} \Xi_{c}^{+}}+a_{\Sigma^{0} \Xi_{c}^{\prime 0}} \frac{m_{\Xi_{c}^{+}}+m_{\Xi_{c}^{\prime 0}}}{m_{\Sigma^{0}}-m_{\Xi_{c}^{\prime 0}}} g_{\Xi_{c}^{\prime 0} \Xi_{c}^{+}}^{A\left(\pi^{+}\right)}\right), \\
& B^{\mathrm{ca}}\left(\Xi_{c}^{+} \rightarrow \Sigma^{+} \pi^{0}\right)=\frac{\sqrt{2}}{f_{\pi}}\left(g_{\Sigma^{+} \Sigma^{+}}^{A\left(\pi^{0}\right)} \frac{m_{\Sigma^{+}}+m_{\Sigma^{+}}}{m_{\Xi_{c}^{+}}-m_{\Sigma^{+}}} a_{\Sigma^{+} \Xi_{c}^{+}}\right), \\
& B^{\mathrm{ca}}\left(\Xi_{c}^{+} \rightarrow \Sigma^{+} \eta_{8}\right)=\frac{\sqrt{2}}{f_{\eta_{8}}}\left(g_{\Sigma^{+} \Sigma^{+}}^{A\left(\eta_{8}\right)} \frac{m_{\Sigma^{+}}+m_{\Sigma^{+}}}{m_{\Xi_{c}^{+}}-m_{\Sigma^{+}}} a_{\Sigma^{+} \Xi_{c}^{+}}+a_{\Sigma^{+} \Xi_{c}^{+}} \frac{m_{\Xi_{c}^{+}}+m_{\Xi_{c}^{+}}}{m_{\Sigma^{+}}-m_{\Xi_{c}^{+}}} g_{\Xi_{c}^{+} \Xi_{c}^{+}}^{A\left(\eta_{8}\right)}+a_{\Sigma^{+} \Xi_{c}^{\prime+}} \frac{m_{\Xi_{c}^{+}}+m_{\Xi_{c}^{\prime+}}}{m_{\Sigma^{+}}-m_{\Xi_{c}^{\prime+}}} g_{\Xi_{c}^{\prime+} \Xi_{c}^{+}}^{A\left(\eta_{8}\right)}\right),
\end{aligned}
$$

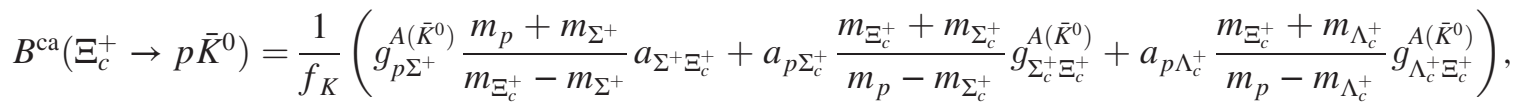

$$
\begin{aligned}
& B^{\mathrm{ca}}\left(\Xi_{c}^{+} \rightarrow \Xi^{0} K^{+}\right)=\frac{1}{f_{K}}\left(g_{\Xi^{0} \Sigma^{+}}^{A\left(K^{+}\right)} \frac{m_{\Xi^{0}}+m_{\Sigma^{+}}}{m_{\Xi_{c}^{+}}-m_{\Sigma^{+}}} a_{\Sigma^{+} \Xi_{c}^{+}}+a_{\Xi^{0} \Omega_{c}^{0}} \frac{m_{\Xi_{c}^{+}}+m_{\Omega_{c}^{0}}}{m_{\Xi^{0}}-m_{\Omega_{c}^{0}}} g_{\Omega_{c}^{0} \Xi_{c}^{+}}^{A\left(K^{+}\right)}\right),
\end{aligned}
$$


and

$$
\begin{aligned}
& B^{\mathrm{ca}}\left(\Xi_{c}^{0} \rightarrow \Lambda \pi^{0}\right)=\frac{\sqrt{2}}{f_{\pi}}\left(g_{\Lambda \Sigma^{0}}^{A\left(\pi^{0}\right)} \frac{m_{\Lambda}+m_{\Sigma^{0}}}{m_{\Xi_{c}^{0}}-m_{\Sigma^{0}}} a_{\Sigma^{0} \Xi_{c}^{0}}+g_{\Lambda \Lambda}^{A\left(\pi^{0}\right)} \frac{m_{\Lambda}+m_{\Lambda}}{m_{\Xi_{c}^{0}}-m_{\Lambda}} a_{\Lambda \Xi_{c}^{0}}\right), \\
& B^{\mathrm{ca}}\left(\Xi_{c}^{0} \rightarrow \Lambda \eta_{8}\right)=\frac{\sqrt{2}}{f_{\eta_{8}}}\left(g_{\Lambda \Sigma^{0}}^{A\left(\eta_{8}\right)} \frac{m_{\Lambda}+m_{\Sigma^{0}}}{m_{\Xi_{c}^{0}}-m_{\Sigma^{0}}} a_{\Sigma^{0} \Xi_{c}^{0}}+g_{\Lambda \Lambda}^{A\left(\eta_{8}\right)} \frac{m_{\Lambda}+m_{\Lambda}}{m_{\Xi_{c}^{0}}-m_{\Lambda}} a_{\Lambda \Xi_{c}^{0}}+a_{\Lambda \Xi_{c}^{0}} \frac{m_{\Xi_{c}^{0}}+m_{\Xi_{c}^{0}}}{m_{\Lambda}-m_{\Xi_{c}^{0}}} g_{\Xi_{c}^{0} \Xi_{c}^{0}}^{A\left(\eta_{8}\right)}+a_{\Lambda \Xi_{c}^{\prime 0}} \frac{m_{\Xi_{c}^{0}}+m_{\Xi_{c}^{\prime 0}}}{m_{\Lambda}-m_{\Xi_{c}^{\prime 0}}} g_{\Xi_{c}^{\prime 0} \Xi_{c}^{0}}^{A\left(\eta_{8}\right)}\right) \\
& B^{\mathrm{ca}}\left(\Xi_{c}^{0} \rightarrow \Sigma^{0} \pi^{0}\right)=\frac{\sqrt{2}}{f_{\pi}}\left(g_{\Sigma^{0} \Sigma^{0}}^{A\left(\pi^{0}\right)} \frac{m_{\Sigma^{0}}+m_{\Sigma^{0}}}{m_{\Xi_{c}^{0}}-m_{\Sigma^{0}}} a_{\Sigma^{0} \Xi_{c}^{0}}+g_{\Sigma^{0} \Lambda}^{A\left(\pi^{0}\right)} \frac{m_{\Sigma^{0}}+m_{\Lambda}}{m_{\Xi_{c}^{0}}-m_{\Lambda}} a_{\Lambda \Xi_{c}^{0}}\right) \\
& B^{\mathrm{ca}}\left(\Xi_{c}^{0} \rightarrow \Sigma^{0} \eta_{8}\right)=\frac{\sqrt{2}}{f_{\eta_{8}}}\left(g_{\Sigma^{0} \Sigma^{0}}^{A\left(\eta_{8}\right)} \frac{m_{\Sigma^{0}}+m_{\Sigma^{0}}}{m_{\Xi_{c}^{0}}-m_{\Sigma^{0}}} a_{\Sigma^{0} \Xi_{c}^{0}}+g_{\Sigma^{0} \Lambda}^{A\left(\eta_{8}\right)} \frac{m_{\Sigma^{0}}+m_{\Lambda}}{m_{\Xi_{c}^{0}}-m_{\Lambda}} a_{\Lambda} \Xi_{c}^{0}+a_{\Sigma^{0} \Xi_{c}^{0}} \frac{m_{\Xi_{c}^{0}}+m_{\Xi_{c}^{0}}}{m_{\Sigma^{0}}-m_{\Xi_{c}^{0}}} g_{\Xi_{c}^{0} \Xi_{c}^{0}}^{A\left(\eta_{8}\right)}+a_{\Sigma^{0} \Xi_{c}^{\prime 0}} \frac{m_{\Xi_{c}^{0}}+m_{\Xi_{c}^{\prime}}}{m_{\Sigma^{0}}-m_{\Xi_{\Xi_{c}^{\prime 0}}}} g_{\Xi_{c}^{\prime} \Xi_{c}^{0}}^{A\left(\eta_{8}\right)}\right), \\
& B^{\mathrm{ca}}\left(\Xi_{c}^{0} \rightarrow \Sigma^{-} \pi^{+}\right)=\frac{1}{f_{\pi}}\left(g_{\Sigma^{-} \Sigma^{0}}^{A\left(\pi^{+}\right)} \frac{m_{\Sigma^{-}}+m_{\Sigma^{0}}}{m_{\Xi_{c}^{0}}-m_{\Sigma^{0}}} a_{\Sigma^{0} \Xi_{c}^{0}}+g_{\Sigma^{-} \Lambda}^{A\left(\pi^{+}\right)} \frac{m_{\Sigma^{-}}+m_{\Lambda}}{m_{\Xi_{c}^{0}}-m_{\Lambda}} a_{\Lambda \Xi_{c}^{0}}\right), \\
& B^{\mathrm{ca}}\left(\Xi_{c}^{0} \rightarrow \Sigma^{+} \pi^{-}\right)=\frac{1}{f_{\pi}}\left(g_{\Sigma^{+} \Sigma^{0}}^{A\left(\pi^{-}\right)} \frac{m_{\Sigma^{+}}+m_{\Sigma^{0}}}{m_{\Xi_{c}^{0}}-m_{\Sigma^{0}}} a_{\Sigma^{0} \Xi_{c}^{0}}+g_{\Sigma^{+} \Lambda}^{A\left(\pi^{-}\right)} \frac{m_{\Sigma^{+}}+m_{\Lambda}}{m_{\Xi_{c}^{0}}-m_{\Lambda}} a_{\Lambda \Xi_{c}^{0}}\right) \text {. } \\
& B^{\mathrm{ca}}\left(\Xi_{c}^{0} \rightarrow p K^{-}\right)=\frac{1}{f_{K}}\left(g_{p \Sigma^{0}}^{A\left(K^{-}\right)} \frac{m_{p}+m_{\Sigma^{0}}}{m_{\Xi_{c}^{0}}-m_{\Sigma^{0}}} a_{\Sigma^{0} \Xi_{c}^{0}}+g_{p \Lambda}^{A\left(K^{-}\right)} \frac{m_{p}+m_{\Lambda}}{m_{\Xi_{c}^{0}}-m_{\Lambda}} a_{\Lambda \Xi_{c}^{0}}\right), \\
& B^{\mathrm{ca}}\left(\Xi_{c}^{0} \rightarrow n \bar{K}^{0}\right)=\frac{1}{f_{K}}\left(g_{n \Sigma^{0}}^{A\left(\bar{K}^{0}\right)} \frac{m_{n}+m_{\Sigma^{0}}}{m_{\Xi_{c}^{0}}-m_{\Sigma^{0}}} a_{\Sigma^{0} \Xi_{c}^{0}}+g_{n \Lambda}^{A\left(\bar{K}^{0}\right)} \frac{m_{n}+m_{\Lambda}}{m_{\Xi_{c}^{0}}-m_{\Lambda}} a_{\Lambda \Xi_{c}^{0}}+a_{n \Sigma_{c}^{0}} \frac{m_{\Xi_{c}^{0}}+m_{\Sigma_{c}^{0}}}{m_{n}-m_{\Sigma_{c}^{0}}} g_{\Sigma_{c}^{\left(\bar{K}^{0}\right.} \Xi_{c}^{0}}\right), \\
& B^{\mathrm{ca}}\left(\Xi_{c}^{0} \rightarrow \Xi^{0} K^{0}\right)=\frac{1}{f_{K}}\left(a_{\Xi^{0} \Omega_{c}^{0}} \frac{m_{\Xi_{c}^{0}}+m_{\Omega_{c}^{0}}}{m_{\Xi^{0}}-m_{\Omega_{c}^{0}}} g_{\Omega_{c}^{0} \Xi_{c}^{0}}^{A\left(K^{0}\right)}+g_{\Xi^{0} \Sigma^{0}}^{A\left(K^{0}\right)} \frac{m_{\Xi^{0}}+m_{\Sigma^{0}}}{m_{\Xi_{c}^{0}}-m_{\Sigma^{0}}} a_{\Sigma^{0} \Xi_{c}^{0}}+g_{\Xi^{0} \Lambda}^{A\left(K^{0}\right)} \frac{m_{\Xi^{0}}+m_{\Lambda}}{m_{\Xi_{c}^{0}}-m_{\Lambda}} a_{\Lambda \Xi_{c}^{0}}\right), \\
& B^{\mathrm{ca}}\left(\Xi_{c}^{0} \rightarrow \Xi^{-} K^{+}\right)=\frac{1}{f_{K}}\left(g_{\Xi^{-} \Sigma^{0}}^{A\left(K^{+}\right)} \frac{m_{\Xi^{-}}+m_{\Sigma^{0}}}{m_{\Xi_{c}^{0}}-m_{\Sigma^{0}}} a_{\Sigma^{0} \Xi_{c}^{0}}+g_{\Xi^{-} \Lambda}^{A\left(K^{+}\right)} \frac{m_{\Xi^{-}}+m_{\Lambda}}{m_{\Xi_{c}^{0}}-m_{\Lambda}} a_{\Lambda \Xi_{c}^{0}}\right) \text {. }
\end{aligned}
$$

The nonfactorizable $P$-wave amplitudes for SCS decays of $\Lambda_{c}^{+}$can be found in [26]. In addition to the baryon matrix element $a_{B B^{\prime}}$, another quantity in the nonfactorizable part of $P$-wave amplitude is the axial-vector form factor $g_{B^{\prime} B}^{A(P)}$. For consistency, the estimation of $g_{\mathcal{B}^{\prime} \mathcal{B}}^{A(P)}$ is carried out in the MIT bag model and the results are shown in Sec. D 2. As seen in the next section, one of the $W$-exchange diagrams, the socalled type-III diagram in which the quark pair is produced between the two quark lines without $W$-exchange, does not contribute to the nonfactorizable $S$ - and $P$-wave amplitudes. This will be discussed in detail there.

\section{NUMERICAL RESULTS AND DISCUSSIONS}

\section{A. $\Lambda_{c}^{+}$decays}

Before proceeding to the $\Xi_{c}$ sector, we first discuss $\Lambda_{c}^{+}$ decays as the measurements of branching fractions and decay asymmetries are well established for many of the channels. The goal is to see what we can learn from the $\Lambda_{c}^{+}$ physics. We show in Table III the results of calculations for $\mathrm{CF}$ and SCS $\Lambda_{c}^{+}$decays. For the form factors $f_{1}$ and $g_{1}$, we follow [39] to use ${ }^{7}$

\footnotetext{
${ }^{7}$ The sign of the form factors is fixed by Eq. (19).
}

$$
f_{1}^{\Lambda_{c} p}(0)=-0.470, \quad g_{1}^{\Lambda_{c} p}(0)=-0.414
$$

for $\Lambda_{c}-p$ transition and rescale the form factors for $\Lambda_{c}-\Lambda$ transition to fit the decay $\Lambda_{c}^{+} \rightarrow \Lambda \pi^{+}$so that $f_{1}^{\Lambda_{c} \Lambda}(0)=0.406$ and $g_{1}^{\Lambda_{c} \Lambda}(0)=0.370 .{ }^{8}$ We see from Table III that the calculated branching fractions and decay asymmetries are in general consistent with experiment except for the decay asymmetry in the decay $\Lambda_{c}^{+} \rightarrow p \bar{K}^{0}$. While all the predictions of $\alpha\left(\Lambda_{c}^{+} \rightarrow p \bar{K}^{0}\right)$ in the literature are all negative except [22], the measured asymmetry by BESIII turns out to be positive with a large uncertainty, $0.18 \pm 0.45$ [30]. This issue needs to be resolved in future study.

We next turn to the mode $\Lambda_{c}^{+} \rightarrow \Xi^{0} K^{+}$, which deserves special attention. It has been shown that its $S$ - and $P$-wave amplitudes are very small due to strong cancellation between various contributions. More specifically (see, e.g., [23]),

\footnotetext{
${ }^{8}$ We have checked if the form factors for $\Lambda_{c}^{+}-p$ and $\Lambda_{c}^{+}-\Lambda$ transitions given in Appendix $\mathrm{C}$ are used, the resulting decay asymmetries will remain stable, but the calculated branching fractions are not as good as those shown in Table III but within a factor of 2 .
} 
TABLE III. The predicted $S$ - and $P$-wave amplitudes of Cabibbo-favored (upper entry) and singly Cabibbo-suppressed (lower entry) $\Lambda_{c}^{+} \rightarrow \mathcal{B}+P$ decays in units of $10^{-2} G_{F} \mathrm{GeV}^{2}$. Branching fractions and the asymmetry parameter $\alpha$ are shown in the last four columns. Experimental results for decay asymmetries are taken from [30] except the modes $\Lambda \pi^{+}$and $\Sigma^{+} \pi^{0}$ where the world averages are obtained from [30] and [7].

\begin{tabular}{lrrrrrrrrrr}
\hline \hline Channel & $A^{\text {fac }}$ & $A^{\text {com }}$ & $A^{\text {tot }}$ & $B^{\text {fac }}$ & $B^{\text {ca }}$ & $B^{\text {tot }}$ & $\mathcal{B}_{\text {theo }}$ & $\mathcal{B}_{\text {exp }}[7]$ & $\alpha_{\text {theo }}$ & $\alpha_{\text {exp }}$ \\
\hline$\Lambda_{c}^{+} \rightarrow p \bar{K}^{0}$ & 3.45 & 4.48 & 7.93 & -6.98 & -2.06 & -9.04 & $2.11 \times 10^{-2}$ & $(3.18 \pm 0.16) 10^{-2}$ & -0.75 & $0.18 \pm 0.45$ \\
$\Lambda_{c}^{+} \rightarrow \Lambda \pi^{+}$ & 5.34 & 0 & 5.34 & -14.11 & 3.60 & -10.51 & $1.30 \times 10^{-2}$ & $(1.30 \pm 0.07) 10^{-2}$ & -0.93 & $-0.84 \pm 0.09$ \\
$\Lambda_{c}^{+} \rightarrow \Sigma^{0} \pi^{+}$ & 0 & 7.68 & 7.68 & 0 & -11.38 & -11.38 & $2.24 \times 10^{-2}$ & $(1.29 \pm 0.07) 10^{-2}$ & -0.76 & $-0.73 \pm 0.18$ \\
$\Lambda_{c}^{+} \rightarrow \Sigma^{+} \pi^{0}$ & 0 & -7.68 & -7.68 & 0 & 11.34 & 11.34 & $2.24 \times 10^{-2}$ & $(1.25 \pm 0.10) 10^{-2}$ & -0.76 & $-0.55 \pm 0.11$ \\
$\Lambda_{c}^{+} \rightarrow \Xi^{0} K^{+}$ & 0 & -4.48 & -4.48 & 0 & -12.10 & -12.10 & $0.73 \times 10^{-2}$ & $(0.55 \pm 0.07) 10^{-2}$ & 0.90 & -0.95 \\
$\Lambda_{c}^{+} \rightarrow \Sigma^{+} \eta$ & 0 & 3.10 & 3.10 & 0 & -15.54 & -15.54 & $0.74 \times 10^{-2}$ & $(0.53 \pm 0.15) 10^{-2}$ & -0.97 \\
$\Lambda_{c}^{+} \rightarrow p \pi^{0}$ & 0.41 & -0.81 & -0.40 & -0.87 & 2.07 & 1.21 & $1.26 \times 10^{-4}$ & $<2.7 \times 10^{-4}$ & -0.97 \\
$\Lambda_{c}^{+} \rightarrow p \eta$ & -0.96 & -1.11 & -2.08 & 1.93 & -0.34 & 1.59 & $1.28 \times 10^{-3}$ & $(1.24 \pm 0.29) 10^{-3}$ & -0.55 \\
$\Lambda_{c}^{+} \rightarrow n \pi^{+}$ & 1.64 & -1.15 & 0.50 & -3.45 & 2.93 & -0.52 & & & \\
$\Lambda_{c}^{+} \rightarrow \Lambda K^{+}$ & 1.66 & -0.08 & 1.58 & -4.43 & 0.55 & -3.70 & $1.07 \times 10^{-3}$ & $(6.1 \pm 1.2) 10^{-4}$ & -0.96 \\
$\Lambda_{c}^{+} \rightarrow \Sigma^{0} K^{+}$ & 0 & 1.49 & 1.49 & 0 & -2.29 & -2.29 & $7.23 \times 10^{-4}$ & $(5.2 \pm 0.8) 10^{-4}$ & -0.73 \\
$\Lambda_{c}^{+} \rightarrow \Sigma^{+} K^{0}$ & 0 & 2.10 & 2.10 & 0 & -3.24 & -3.24 & $1.44 \times 10^{-3}$ & & $\cdots$ & -0.73 \\
\hline \hline
\end{tabular}

$$
\begin{aligned}
A^{\mathrm{com}}\left(\Lambda_{c}^{+} \rightarrow \Xi^{0} K^{+}\right)= & \frac{1}{f_{K}}\left(a_{\Sigma^{+} \Lambda_{c}^{+}}-a_{\Xi^{0} \Xi_{c}^{0}}\right), \\
B^{\mathrm{ca}}\left(\Lambda_{c}^{+} \rightarrow \Xi^{0} K^{+}\right)= & \frac{1}{f_{K}}\left(g_{\Xi^{0} \Sigma^{+}}^{A\left(K^{+}\right)} \frac{m_{\Xi^{0}}+m_{\Sigma^{+}}}{m_{\Lambda_{c}^{+}}-m_{\Sigma^{+}}} a_{\Sigma^{+} \Lambda_{c}^{+}}\right. \\
& +a_{\Xi^{0} \Xi_{c}^{0}} \frac{m_{\Xi_{c}^{0}}+m_{\Lambda_{c}^{+}}}{m_{\Xi^{0}}-m_{\Xi_{c}^{0}}} g_{\Xi_{c}^{0} \Lambda_{c}^{+}} \\
& \left.+a_{\Xi^{0} \Xi_{c}^{\prime 0}} \frac{m_{\Xi_{c}^{\prime 0}}+m_{\Lambda_{c}^{+}}}{m_{\Xi^{0}}-m_{\Xi_{c}^{\prime 0}}} g_{\Xi_{c}^{\prime \prime} \Lambda_{c}^{+}}^{A\left(K^{+}\right)}\right) .
\end{aligned}
$$

Since the matrix elements $a_{\Sigma^{+}} \Lambda_{c}^{+}$and $a_{\Xi^{0} \Xi_{c}^{0}}$ are identical in the $\mathrm{SU}(3)$ limit and since there is a large cancellation between the first and third terms in $B^{\text {ca }}$ (no contribution from the second term due to the vanishing $g_{\Xi_{c}^{0} \Lambda_{c}^{+}}^{A\left(K^{+}\right)}$; for details see [23]), the calculated branching fraction turns out to be too small compared to experiment and the decay asymmetry is predicted to be 0 owing to the vanishing $S$-wave amplitude [20-22,24,25]. This is a long-standing puzzle.

To solve the above-mentioned puzzle, we notice that one of the $W$-exchange diagrams depicted in the left panel of Fig. 1(a) can be described by two distinct pole diagrams at the hadron level shown in the right panel of the diagram 1(a). These two pole diagrams are called type-III diagrams in [20] and (d1) and (d2) in [24]. As first pointed out by Körner and Krämer [20], the type-III diagram contributes only to the $P$-wave amplitude. Moreover, they pointed out that this diagram is empirically observed to be
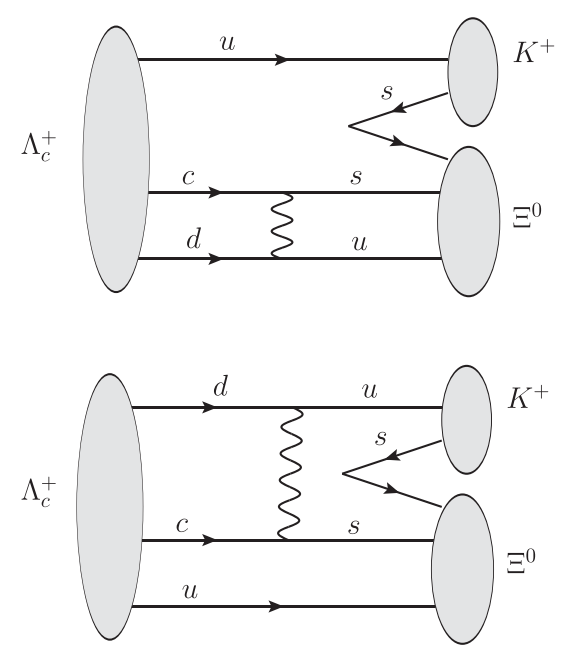

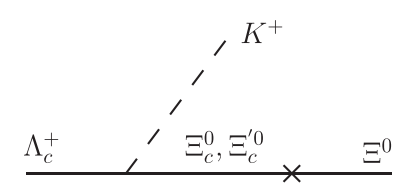

(a)

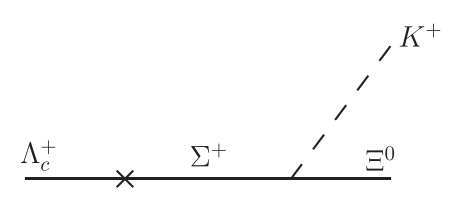

(b)

a)

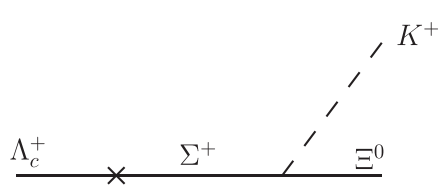


TABLE IV. The Cabibbo-favored decays $\Xi_{c} \rightarrow \mathcal{B}_{f} P$ in units of $10^{-2} G_{F} \mathrm{GeV}^{2}$. Branching fractions (in percent) and the up-down decay asymmetry $\alpha$ in theory and experiment are shown in the last four columns. Experimental results are taken from [9-11] for branching fractions and [41] for decay asymmetry.

\begin{tabular}{lcccccccccc}
\hline \hline Channel & $A^{\text {fac }}$ & $A^{\text {com }}$ & $A^{\text {tot }}$ & $B^{\text {fac }}$ & $B^{\text {ca }}$ & $B^{\text {tot }}$ & $\mathcal{B}_{\text {theo }}$ & $\mathcal{B}_{\text {exp }}$ & $\alpha_{\text {theo }}$ & $\alpha_{\text {exp }}$ \\
\hline$\Xi_{c}^{+} \rightarrow \Sigma^{+} \bar{K}^{0}$ & 2.98 & -4.48 & -1.50 & -9.95 & 12.28 & 2.32 & 0.20 & $\ldots$ & -0.80 & $\ldots$ \\
$\Xi_{c}^{+} \rightarrow \Xi^{0} \pi^{+}$ & -7.41 & 5.36 & -2.05 & 28.07 & -14.03 & 14.04 & 1.72 & $1.57 \pm 0.83$ & -0.78 & $\ldots$ \\
$\Xi_{c}^{0} \rightarrow \Lambda \bar{K}^{0}$ & -1.11 & -5.41 & -6.52 & 3.66 & 6.87 & 10.52 & 1.33 & $\ldots$ & -0.86 & $\ldots$ \\
$\Xi_{c}^{0} \rightarrow \Sigma^{0} \bar{K}^{0}$ & -2.11 & 3.12 & 1.02 & 7.05 & -9.39 & -2.33 & 0.04 & $\ldots$ & -0.96 & $\ldots$ \\
$\Xi_{c}^{0} \rightarrow \Sigma^{+} K^{-}$ & 0 & -4.42 & -4.42 & 0 & -11.32 & -11.32 & 0.78 & $\ldots$ & 0.98 & $\ldots$ \\
$\Xi_{c}^{0} \rightarrow \Xi^{0} \pi^{0}$ & 0 & -7.58 & -7.58 & 0 & 11.79 & 11.79 & 1.82 & $\ldots$ & -0.77 & $\ldots$ \\
$\Xi_{c}^{0} \rightarrow \Xi^{0} \eta$ & 0 & -10.80 & -10.80 & 0 & -6.17 & -6.17 & 2.67 & $\ldots$ & 0.30 & $\ldots$ \\
$\Xi_{c}^{0} \rightarrow \Xi^{-} \pi^{+}$ & -7.42 & -5.36 & -12.78 & 28.24 & 2.65 & 30.89 & 6.47 & $1.80 \pm 0.52$ & -0.95 & $-0.6 \pm 0.4$ \\
\hline \hline
\end{tabular}

strongly suppressed. It was argued by Żenczykowski [24] that contributions from diagrams (d1) and (d2) cancel each other due to the spin-flavor structure. Hence, its $S$ - and $P$-wave amplitudes vanish. The smallness of type-III $W$ exchange diagram also can be numerically checked through Eq. (40). In other words, the conventional expression of parity-violating and -conserving amplitudes given in Eq. (40) is actually for the type-III $W$-exchange diagram in Fig. 1(a). As a result, nonvanishing nonfactorizable $S$ - and $P$-wave amplitudes arise solely from the $W$-exchange diagram depicted in Fig. 1(b) (called the type-II $W$-exchange diagram in [20] and (b)-type diagram in [24]). The nonfactorizable amplitudes induced from type-II $W$-exchange now read

$$
\begin{aligned}
A^{\mathrm{com}}\left(\Lambda_{c}^{+} \rightarrow \Xi^{0} K^{+}\right) & =\frac{1}{f_{K}} a_{\Sigma^{+} \Lambda_{c}^{+}}, \\
B^{\mathrm{ca}}\left(\Lambda_{c}^{+} \rightarrow \Xi^{0} K^{+}\right) & =\frac{1}{f_{K}}\left(g_{\Xi^{0} \Sigma^{+}}^{A\left(K^{+}\right)} \frac{m_{\Xi^{0}}+m_{\Sigma^{+}}}{m_{\Lambda_{c}^{+}}-m_{\Sigma^{+}}} a_{\Sigma^{+}} \Lambda_{c}^{+}\right) .
\end{aligned}
$$

Consequently, both partial wave amplitudes are not subject to large cancellations.

Note that the pole diagram induced by type-II $W$ exchange is the same as the second pole diagram (i.e., a weak transition of $\Lambda_{c}^{+}-\Sigma^{+}$followed by a strong emission of $K^{+}$) in Fig. 1(a), but it is no longer canceled by the first pole diagram. A vanishing $S$-wave amplitude was often claimed in the literature. We stress again that the parity-violating amplitude can be induced from type-II $W$-exchange through current algebra. ${ }^{9}$ Equation (41) leads to $\mathcal{B}\left(\Lambda_{c}^{+} \rightarrow \Xi^{0} K^{+}\right)=0.71 \%$, which is consistent with the data of $(0.55 \pm 0.07) \%$ [7]. Moreover, the predicted positive decay asymmetry of order 0.90 is consistent with

\footnotetext{
${ }^{9}$ It had been argued that the contribution from type-II diagrams to the $S$-wave amplitude of $\Lambda_{c}^{+} \rightarrow \Xi^{0} K^{+}$vanishes based on SU(4) symmetry $[20,24]$. This is no longer true in the presence of $\mathrm{SU}(4)$-symmetry breaking.
}

the value of $\alpha\left(\Lambda_{c}^{+} \rightarrow \Xi^{0} K^{+}\right)=0.94_{-0.11}^{+0.06}$ obtained in the SU(3)-flavor approach [15]. ${ }^{10}$ Therefore, the long-standing puzzle with the branching fraction and the decay asymmetry of $\Lambda_{c}^{+} \rightarrow \Xi^{0} K^{+}$is resolved.

In the $\Xi_{c}$ sector, vanishing type-III $W$-exchange contributions also occur in the CF decay $\Xi_{c}^{0} \rightarrow \Sigma^{+} K^{-}$and the SCS modes $\Xi_{c}^{0} \rightarrow p K^{-}, \Sigma^{+} \pi^{-}$. We come to this point later.

Comparing Table III with Table II of [26] for SCS $\Lambda_{c}^{+}$ decays, we see some changes in the $P$-wave amplitudes of $\Lambda_{c}^{+} \rightarrow p \pi^{0}, p \eta, n \pi^{+}$. This is because the first equation in (C2) of [26] should read

$$
g_{n p}^{A\left(\pi^{+}\right)}=2 g_{p p}^{A\left(\pi^{0}\right)}=\frac{10}{\sqrt{3}} g_{p p}^{A\left(\eta_{8}\right)}=\frac{5}{3}\left(4 \pi Z_{1}\right) .
$$

Consequently, we find $\mathcal{B}\left(\Lambda_{c}^{+} \rightarrow p \pi^{0}\right)$ is modified from $0.75 \times 10^{-4}$ [26] to the current value of $1.26 \times 10^{-4}$. As for $\Lambda_{c}^{+} \rightarrow n \pi^{+}$, after correcting the error with the axial-vector form factor $g_{\Sigma_{c}^{0} \Lambda_{c}}^{A\left(\pi^{+}\right)}$we find large cancellation in both $S$ - and $P$-wave amplitudes, resulting in a very small branching fraction of order $0.9 \times 10^{-4}$. Since the large cancellation renders the present theoretical predictions of $\Lambda_{c}^{+} \rightarrow n \pi^{+}$ unreliable, we do not show its branching fraction and decay asymmetry in Tables III and VI.

\section{B. $\Xi_{c}$ decays}

Branching fractions and up-down decay asymmetries of CF and SCS $\Xi_{c}^{+, 0}$ weak decays are calculated according to Eqs. (5)-(7), yielding the numerical results shown in Tables IV and V, respectively. One interesting point is that there does not exist any decay mode that proceeds only through the factorizable diagram. Among all the processes, the three modes $\Xi_{c}^{0} \rightarrow \Sigma^{+} K^{-}, \Xi^{0} \pi^{0}, \Xi^{0} \eta_{8}$ in CF processes and the five SCS modes $\Xi_{c}^{+} \rightarrow p \bar{K}^{0}, \Xi_{c}^{0} \rightarrow \Xi^{0} K^{0}, p K^{-}$,

\footnotetext{
${ }^{10} \mathrm{By}$ measuring the angular dependence $1+\alpha_{\Xi K} \cos ^{2} \theta_{K}$ in the process $\Lambda_{c}^{+} \rightarrow \Xi^{0} K^{+}$, BESIII obtained $\alpha_{\Xi K}=0.77 \pm 0.78$ [40]. However, this quantity should not be confused with the decay asymmetry $\alpha\left(\Lambda_{c}^{+} \rightarrow \Xi^{0} K^{+}\right)$, which is yet to be measured.
} 
TABLE V. The singly Cabibbo-suppressed decays $\Xi_{c} \rightarrow \mathcal{B}_{f} P$ in units of $10^{-2} G_{F} \mathrm{GeV}^{2}$. Branching fractions (in unit of $10^{-3}$ ) and the asymmetry parameter $\alpha$ are shown in the last two columns.

\begin{tabular}{|c|c|c|c|c|c|c|c|c|}
\hline Channel & $A^{\mathrm{fac}}$ & $A^{\mathrm{com}}$ & $A^{\text {tot }}$ & $B^{\mathrm{fac}}$ & $B^{\mathrm{ca}}$ & $B^{\text {tot }}$ & $\mathcal{B}_{\text {theo }}$ & $\alpha_{\text {theo }}$ \\
\hline$\Xi_{c}^{+} \rightarrow \Lambda \pi^{+}$ & 0.46 & -1.50 & -1.04 & -1.69 & 2.16 & 0.47 & 0.85 & -0.33 \\
\hline$\Xi_{c}^{+} \rightarrow \Sigma^{0} \pi^{+}$ & -0.90 & -1.00 & -1.90 & 3.29 & 0.74 & 4.03 & 4.30 & -0.95 \\
\hline$\Xi_{c}^{+} \rightarrow \Sigma^{+} \pi^{0}$ & 0.32 & 1.00 & 1.32 & -1.16 & 1.61 & 0.44 & 1.36 & 0.23 \\
\hline$\Xi_{c}^{+} \rightarrow \Sigma^{+} \eta$ & -0.74 & 1.42 & 0.68 & 2.58 & -2.19 & 0.39 & 0.32 & 0.36 \\
\hline$\Xi_{c}^{+} \rightarrow p \bar{K}^{0}$ & 0 & -2.10 & -2.10 & 0 & 2.64 & 2.64 & 3.96 & -0.83 \\
\hline$\Xi_{c}^{+} \rightarrow \Xi^{0} K^{+}$ & -2.30 & 1.16 & -1.14 & 8.43 & -3.46 & 4.97 & 2.20 & -0.98 \\
\hline$\Xi_{c}^{0} \rightarrow \Lambda \pi^{0}$ & -0.12 & 1.06 & 0.95 & 0.42 & -0.96 & -0.53 & 0.24 & -0.41 \\
\hline$\Xi_{c}^{0} \rightarrow \Lambda \eta$ & 0.27 & 1.51 & 1.78 & -0.94 & -0.71 & -1.65 & 0.81 & -0.59 \\
\hline$\Xi_{c}^{0} \rightarrow \Sigma^{0} \pi^{0}$ & -0.23 & -0.70 & -0.93 & 0.82 & 1.36 & 2.18 & 0.38 & -0.98 \\
\hline$\Xi_{c}^{0} \rightarrow \Sigma^{0} \eta$ & 0.53 & -1.01 & -0.48 & -1.83 & 1.55 & -0.28 & 0.05 & 0.36 \\
\hline$\Xi_{c}^{0} \rightarrow \Sigma^{-} \pi^{+}$ & -1.28 & -1.41 & -2.69 & 4.67 & 0.22 & 4.89 & 2.62 & -0.90 \\
\hline$\Xi_{c}^{0} \rightarrow \Sigma^{+} \pi^{-}$ & 0 & 1.41 & 1.41 & 0 & 2.49 & 2.49 & 0.71 & 0.89 \\
\hline$\Xi_{c}^{0} \rightarrow p K^{-}$ & 0 & -0.94 & -0.94 & 0 & -1.86 & -1.86 & 0.35 & 0.99 \\
\hline$\Xi_{c}^{0} \rightarrow n \bar{K}^{0}$ & 0 & -2.10 & -2.10 & 0 & 2.96 & 2.96 & 1.40 & -0.89 \\
\hline$\Xi_{c}^{0} \rightarrow \Xi^{0} K^{0}$ & 0 & 2.10 & 2.10 & 0 & -4.17 & -4.17 & 1.32 & -0.85 \\
\hline$\Xi_{c}^{0} \rightarrow \Xi^{-} K^{+}$ & -2.31 & -0.94 & -3.24 & 8.49 & 0.71 & 9.20 & 3.90 & -0.97 \\
\hline
\end{tabular}

$n \bar{K}^{0}, \quad \Sigma^{+} \pi^{-}$proceed only through the nonfactorizable diagrams, while all the other channels receive contributions from both factorizable and nonfactorizable terms. The relative sign between factorizable and nonfactorizable contributions determines whether the interference term is destructive or constructive. For example, factorizable and nonfactorizable terms in both the $S$ - and $P$-wave amplitudes of the decays $\Xi_{c}^{+} \rightarrow \Sigma^{+} \bar{K}^{0}, \Xi^{0} \pi^{+}$ and $\Xi_{c}^{0} \rightarrow \Sigma^{0} \bar{K}^{0}$ interfere destructively, leading to small branching fractions, especially for the last mode. On the contrary, interference in the channels $\Xi_{c}^{0} \rightarrow \Lambda \bar{K}^{0}, \Xi^{-} \pi^{+}$ is found to be constructive.

The CF decay $\Xi_{c}^{0} \rightarrow \Sigma^{+} K^{-}$and the SCS modes $\Xi_{c}^{0} \rightarrow$ $p K^{-}, \Sigma^{+} \pi^{-}$are of special interest among all the $\Xi_{c}$ weak decays. Their naive $S$-wave amplitudes are given by

$$
\begin{aligned}
A^{\mathrm{com}}\left(\Xi_{c}^{0} \rightarrow \Sigma^{+} K^{-}\right) & =\frac{1}{f_{K}}\left(a_{\Xi^{0} \Xi_{c}^{0}}-a_{\Sigma^{+} \Lambda_{c}^{+}}\right), \\
A^{\mathrm{com}}\left(\Xi_{c}^{0} \rightarrow p K^{-}\right) & =-\frac{1}{f_{K}}\left(\frac{\sqrt{2}}{2} a_{\Sigma^{0} \Xi_{c}^{0}}+\frac{\sqrt{6}}{2} a_{\Lambda \Xi_{c}^{0}}+a_{p \Lambda_{c}^{+}}\right), \\
A^{\mathrm{com}}\left(\Xi_{c}^{0} \rightarrow \Sigma^{+} \pi^{-}\right) & =-\frac{1}{f_{\pi}}\left(\sqrt{2} a_{\Sigma^{0} \Xi_{c}^{0}}+a_{\Sigma^{+} \Xi_{c}^{+}}\right) .
\end{aligned}
$$

From Eqs. (D2) and (D3) for baryon matrix elements, it is easily seen that they all vanish in the SU(3) limit. Likewise, their $P$-wave amplitudes are also subject to large cancellations. Just as for the decay $\Lambda_{c}^{+} \rightarrow \Xi^{0} K^{+}$discussed in Sec. III A, we should neglect the contributions from type-III $W$-exchange diagrams and focus on type-II $W$ exchange ones. The resulting amplitudes for these three modes now read

$$
\begin{aligned}
A^{\operatorname{com}}\left(\Xi_{c}^{0} \rightarrow \Sigma^{+} K^{-}\right) & =\frac{1}{f_{K}} a_{\Xi^{0} \Xi_{c}^{0}}, \\
A^{\operatorname{com}}\left(\Xi_{c}^{0} \rightarrow p K^{-}\right) & =-\frac{1}{f_{K}}\left(\frac{\sqrt{2}}{2} a_{\Sigma^{0} \Xi_{c}^{0}}+\frac{\sqrt{6}}{2} a_{\Lambda \Xi_{c}^{0}}\right), \\
A^{\operatorname{com}}\left(\Xi_{c}^{0} \rightarrow \Sigma^{+} \pi^{-}\right) & =-\frac{\sqrt{2}}{f_{\pi}} a_{\Sigma^{0} \Xi_{c}^{0}},
\end{aligned}
$$

for $S$ wave [see Eqs. (32) and (34)] and Eqs. (36) and (38) for $P$ wave. From Tables IV and V we see that

$\alpha\left(\Xi_{c}^{0} \rightarrow \Sigma^{+} K^{-}\right) \approx 0.98, \quad \alpha\left(\Xi_{c}^{0} \rightarrow p K^{-}\right) \approx 0.99$,

$\alpha\left(\Xi_{c}^{0} \rightarrow \Sigma^{+} \pi^{-}\right) \approx 0.89$.

Hence, their decay asymmetries are all positive and close to unity. It is interesting to notice that the decay asymmetries of these three modes are also predicted to be positive and large in the SU(3) approach of [15].

Besides the above-mentioned three modes of $\Xi_{c}^{0}$, the type-III $W$-exchange diagram also exists in the following channels: $\Xi_{c}^{+} \rightarrow \Xi^{0} K^{+}, \Sigma^{+}\left(\pi^{0}, \eta\right)$ and $\Xi_{c}^{0} \rightarrow\left(\Lambda, \Sigma^{0}\right) \pi^{0}$, $\left(\Lambda, \Sigma^{0}\right) \eta$. However, the effects of vanishing type-III $W$ exchange can be seen only in the $P$-wave amplitudes of $\Xi_{c}^{+} \rightarrow \Sigma^{+} \pi^{0}$ and $\Xi_{c}^{0} \rightarrow\left(\Lambda, \Sigma^{0}\right) \pi^{0}$. In Eqs. (37) and (38) for these three modes we have explicitly dropped the pole contributions with the strong $\pi^{0}$ emission from $\Xi_{c}$ followed by a weak transition.

As for the two modes $\Xi_{c}^{+} \rightarrow \Xi^{0} \pi^{+}$and $\Xi_{c}^{0} \rightarrow \Xi^{-} \pi^{+}$, we see from Table IV that our prediction is in good agreement with experiment for the former, but it is too large compared to the experimental measurement for the latter. This is mainly due to the relative sign between factorizable and nonfactorizable terms. In the absence of nonfactorizable 
TABLE VI. Comparison of this work with [15,48] for the branching fractions in units of $10^{-2}$ for Cabibbo-favored $\Lambda_{c}^{+}$decays (upper entry) and $10^{-3}$ for singly Cabibbo-suppressed ones (lower entry). Decay asymmetries are shown in parentheses.

\begin{tabular}{lccr}
\hline \hline Modes & This work & Geng et al. $[15,48]$ & Experiment \\
\hline$\Lambda_{c}^{+} \rightarrow \Lambda \pi^{+}$ & $1.30(-0.93)$ & $1.27 \pm 0.07(-0.77 \pm 0.07)$ & $1.30 \pm 0.07(-0.84 \pm 0.09)$ \\
$\Lambda_{c}^{+} \rightarrow \Sigma^{0} \pi^{+}$ & $2.24(-0.76)$ & $1.26 \pm 0.06(-0.58 \pm 0.10)$ & $1.29 \pm 0.07(-0.73 \pm 0.18)$ \\
$\Lambda_{c}^{+} \rightarrow \Sigma^{+} \pi^{0}$ & $2.24(-0.76)$ & $1.26 \pm 0.06(-0.58 \pm 0.10)$ & $1.25 \pm 0.10(-0.55 \pm 0.11)$ \\
$\Lambda_{c}^{+} \rightarrow \Sigma^{+} \eta$ & $0.74(-0.95)$ & $0.29 \pm 0.12\left(-0.70_{-0.30}^{+0.59}\right)$ & $0.53 \pm 0.15$ \\
$\Lambda_{c}^{+} \rightarrow p \bar{K}^{0}$ & $2.11(-0.75)$ & $3.14 \pm 0.15\left(-0.99_{-0.01}^{+0.09}\right)$ & $3.18 \pm 0.16(0.18 \pm 0.45)$ \\
$\Lambda_{c}^{+} \rightarrow \Xi^{0} K^{+}$ & $0.73(0.90)$ & $0.57 \pm 0.09\left(1.00_{-0.02}^{+0.00}\right)$ & $0.55 \pm 0.07(0.77 \pm 0.78)$ \\
$\Lambda_{c}^{+} \rightarrow p \pi^{0}$ & $0.13(-0.97)$ & $0.11_{-0.11}^{+0.13}(0.24 \pm 0.68)$ & $<0.27$ \\
$\Lambda_{c}^{+} \rightarrow p \eta$ & $1.28(-0.55)$ & $1.12 \pm 0.28\left(-1.00_{-0.00}^{+0.06}\right)$ & $1.24 \pm 0.29$ \\
$\Lambda_{c}^{+} \rightarrow n \pi^{+}$ & & $0.76 \pm 0.11(0.27 \pm 0.11)$ & $0.61 \pm 0.12$ \\
$\Lambda_{c}^{+} \rightarrow \Lambda K^{+}$ & $1.07(-0.96)$ & $0.66 \pm 0.09(0.09 \pm 0.26)$ & $0.52 \pm 0.08$ \\
$\Lambda_{c}^{+} \rightarrow \Sigma^{0} K^{+}$ & $0.72(-0.73)$ & $0.52 \pm 0.07\left(-0.98_{-0.02}^{+0.05}\right)$ & $1.05 \pm 0.14\left(-0.98_{-0.02}^{+0.05}\right)$ \\
$\Lambda_{c}^{+} \rightarrow \Sigma^{+} K^{0}$ & $1.44(-0.73)$ & & \\
\hline \hline
\end{tabular}

contributions, we find $\mathcal{B}\left(\Xi_{c}^{+} \rightarrow \Xi^{0} \pi^{+}\right) \approx 9.9 \%$ and $\mathcal{B}\left(\Xi_{c}^{0} \rightarrow \Xi^{-} \pi^{+}\right) \approx 3.3 \%$. Since the measured branching fractions are $(1.57 \pm 0.83) \%$ and $(1.80 \pm 0.52) \%$, respectively, this implies that there should be a large destructive interference between factorizable and nonfactorizable terms in the former and a smaller destructive interference in the latter. We notice that the factorizable amplitudes of these two modes are very similar. ${ }^{11}$ From Eq. (32), it is clear that the commutator terms of both modes denoted by $A^{\text {com }}$ are the same in magnitude but opposite in sign. Consequently, the interference between $A^{\text {fact }}$ and $A^{\text {com }}$ is destructive in $\Xi_{c}^{+} \rightarrow \Xi^{0} \pi^{+}$but constructive in $\Xi_{c}^{0} \rightarrow \Xi^{-} \pi^{+}$ (see also [22]). As a result, the predicted branching fraction of order $6.5 \%$ for the latter is too large. If we use the form factors $f_{1}^{\Xi_{c} \Xi}(0)=-0.590$ and $g_{1}^{\Xi_{c} \Xi}(0)=-0.582[35]$ in conjunction with the $q^{2}$ dependence given by Eq. (18), the branching fraction is reduced only slightly from $6.5 \%$ to $6.2 \%$. Hence, we conclude that these two modes cannot be simultaneously explained within the framework of current algebra for $S$-wave amplitudes.

To circumvent the difficulty with $\Xi_{c}^{0} \rightarrow \Xi^{-} \pi^{+}$, one possibility is to consider the correction to the current-algebra calculation of the parity-violating amplitude by writing

$$
A=A^{\mathrm{CA}}+\left(A-A^{\mathrm{CA}}\right),
$$

where the term $\left(A-A^{\mathrm{CA}}\right)$ can be regarded as an on-shell correction to the current-algebra result. It turns out that in the existing pole model calculations [22,23,34], the on-shell correction $\left(A-A^{\mathrm{CA}}\right)$ always has a sign opposite to that of $A^{\mathrm{CA}}$. Moreover, the on-shell correction is sometimes large

\footnotetext{
${ }^{11}$ We have confirmed that the sign of the factorizable contribution in the earlier work of [23] has to be flipped due to the sign convention with the form factors $f_{1}$ and $g_{1}$.
}

enough to flip the sign of the parity-violating amplitudes. It is conceivable that on-shell corrections could be large for $\Xi^{-} \pi^{+}$but small for $\Xi^{0} \pi^{+}$. This issue needs to be clarified in the future. Nevertheless, we have learned from Table III that current algebra generally works well in $\Lambda_{c}^{+} \rightarrow \mathcal{B}+P$ decays,

For the up-down decay asymmetry, there has been only one measurement thus far. In 2001, the CLEO collaboration measured $\Xi_{c}^{0} \rightarrow \Xi^{-} \pi^{+}$and found $\alpha\left(\Xi_{c}^{0} \rightarrow \Xi^{-} \pi^{+}\right)=-0.6 \pm 0.4$ [41]. Our prediction is consistent with CLEO's value. Decay asymmetries are usually negative in most of the channels. However, besides the three modes $\Xi_{c}^{0} \rightarrow \Sigma^{+} K^{-}$, $p K^{-}, \Sigma^{+} \pi^{-}$as discussed before, the following channels $\Xi_{c}^{0} \rightarrow \Xi^{0} \eta, \Sigma^{0} \eta$ and $\Xi_{c}^{+} \rightarrow \Sigma^{+} \pi^{0}, \Sigma^{+} \eta$ in the $\Xi_{c}$ sector are also predicted to have positive decay asymmetries (see Tables IV and V). We hope that these predictions could be tested in the near future by Belle/Belle II.

\section{Comparison with the SU(3) approach}

Besides dynamical model calculations, two-body nonleptonic decays of charmed baryons have been analyzed in terms of $\mathrm{SU}(3)$-irreducible-representation amplitudes $[42,43]$. There are two distinct approaches to implement this idea. One is to write down the SU(3)-irreduciblerepresentation amplitudes by decomposing the effective Hamiltonian through the Wigner-Eckart theorem. The other is to use the topological quark diagrams that are related in different decay channels via $\mathrm{SU}(3)$ flavor symmetry. Each approach has its own advantage. A general formulation of the quark-diagram scheme for charmed baryons is given in [44] (see also [45]). Analysis of Cabibbosuppressed decays using SU(3) flavor symmetry was first carried out in [46]. This approach became very popular recently [13-15,47]. Although $\mathrm{SU}(3)$ flavor symmetry is approximate, it does provide very useful information. 
TABLE VII. Comparison of this work with $[15,48]$ for the branching fractions in units of $10^{-2}$ for Cabibbo-favored $\Xi_{c}^{+, 0}$ decays (upper entry) and $10^{-3}$ for singly Cabibbo-suppressed ones (lower entry). Decay asymmetries are shown in parentheses. Experimental results are taken from [9-11] for branching fractions and [41] for decay asymmetry.

\begin{tabular}{|c|c|c|c|}
\hline Modes & This work & Geng et al. $[15,48]$ & Experiment \\
\hline$\Xi_{c}^{+} \rightarrow \Sigma^{+} \bar{K}^{0}$ & $0.20(-0.80)$ & $0.78_{-0.78}^{+1.02}\left(0.93_{-0.14}^{+0.07}\right)$ & \\
\hline$\Xi_{c}^{+} \rightarrow \Xi^{0} \pi^{+}$ & $1.72(-0.78)$ & $0.42 \pm 0.17(-0.43 \pm 0.57)$ & $1.57 \pm 0.83$ \\
\hline$\Xi_{c}^{0} \rightarrow \Lambda \bar{K}^{0}$ & $1.33(-0.86)$ & $1.42 \pm 0.09\left(-0.85_{-0.15}^{+0.16}\right)$ & \\
\hline$\Xi_{c}^{0} \rightarrow \Sigma^{0} \bar{K}^{0}$ & $0.04(-0.94)$ & $0.09_{-0.09}^{+0.11}\left(0.30_{-0.84}^{+0.70}\right)$ & \\
\hline$\Xi_{c}^{0} \rightarrow \Sigma^{+} K^{-}$ & $0.78(0.98)$ & $0.76 \pm 0.14\left(0.93_{-0.08}^{+0.07}\right)$ & \\
\hline$\Xi_{c}^{0} \rightarrow \Xi^{0} \pi^{0}$ & $1.82(-0.77)$ & $1.00 \pm 0.14\left(-0.96_{-0.04}^{+0.05}\right)$ & \\
\hline$\Xi_{c}^{0} \rightarrow \Xi^{0} \eta$ & $2.67(0.30)$ & $1.30 \pm 0.23(0.80 \pm 0.16)$ & \\
\hline$\Xi_{c}^{0} \rightarrow \Xi^{-} \pi^{+}$ & $6.47(-0.95)$ & $2.95 \pm 0.14\left(-1.00_{-0.00}^{+0.01}\right)$ & $1.80 \pm 0.52(-0.6 \pm 0.4)$ \\
\hline$\Xi_{c}^{+} \rightarrow \Lambda \pi^{+}$ & $0.85(-0.33)$ & $1.23 \pm 0.42(0.03 \pm 0.18)$ & \\
\hline$\Xi_{c}^{+} \rightarrow \Sigma^{0} \pi^{+}$ & $4.30(-0.95)$ & $2.65 \pm 0.25(-0.61 \pm 0.12)$ & \\
\hline$\Xi_{c}^{+} \rightarrow \Sigma^{+} \pi^{0}$ & $1.36(0.23)$ & $2.61 \pm 0.67(-0.18 \pm 0.36)$ & \\
\hline$\Xi_{c}^{+} \rightarrow \Sigma^{+} \eta$ & $0.32(0.36)$ & $1.50 \pm 1.06(0.30 \pm 0.60)$ & \\
\hline$\Xi_{c}^{+} \rightarrow p \bar{K}^{0}$ & $3.96(-0.83)$ & $4.64 \pm 0.72(-0.83 \pm 0.06)$ & \\
\hline$\Xi_{c}^{+} \rightarrow \Xi^{0} K^{+}$ & $2.20(-0.98)$ & $0.76 \pm 0.12(0.39 \pm 0.16)$ & \\
\hline$\Xi_{c}^{0} \rightarrow \Lambda \pi^{0}$ & $0.24(-0.41)$ & $0.31 \pm 0.11(0.08 \pm 0.22)$ & \\
\hline$\Xi_{c}^{0} \rightarrow \Lambda \eta$ & $0.81(-0.59)$ & $0.79 \pm 0.27(-0.17 \pm 0.26)$ & \\
\hline$\Xi_{c}^{0} \rightarrow \Sigma^{0} \pi^{0}$ & $0.38(-0.98)$ & $0.50 \pm 0.09(-0.74 \pm 0.25)$ & \\
\hline$\Xi_{c}^{0} \rightarrow \Sigma^{0} \eta$ & $0.05(0.36)$ & $0.18 \pm 0.11(-0.20 \pm 0.76)$ & \\
\hline$\Xi_{c}^{0} \rightarrow \Sigma^{-} \pi^{+}$ & $2.62(-0.90)$ & $1.83 \pm 0.09(-0.99 \pm 0.01)$ & \\
\hline$\Xi_{c}^{0} \rightarrow \Sigma^{+} \pi^{-}$ & $0.71(0.89)$ & $0.49 \pm 0.09(0.91 \pm 0.09)$ & \\
\hline$\Xi_{c}^{0} \rightarrow p K^{-}$ & $0.35(0.99)$ & $0.60 \pm 0.13(0.82 \pm 0.11)$ & \\
\hline$\Xi_{c}^{0} \rightarrow n \bar{K}^{0}$ & $1.40(-0.89)$ & $1.07 \pm 0.06(-0.74 \pm 0.12)$ & \\
\hline$\Xi_{c}^{0} \rightarrow \Xi^{0} K^{0}$ & $1.32(-0.85)$ & $0.96 \pm 0.04(-0.53 \pm 0.09)$ & \\
\hline$\Xi_{c}^{0} \rightarrow \Xi^{-} K^{+}$ & $3.90(-0.97)$ & $1.28 \pm 0.06\left(-1.00_{-0.00}^{+0.01}\right)$ & \\
\hline
\end{tabular}

In Tables VI and VII we compare our results for $\Lambda_{c}^{+}$and $\Xi_{c}^{+, 0}$ decays, respectively, with the $S U(3)_{F}$ approach in $[15,48]$ in which the parameters for both $S$ - and $P$-wave amplitudes are obtained by fitting to the data. ${ }^{12}$

We see from Table VI that it appears the SU(3) approach gives a better description of the measured branching fractions because it fits to the data. However, it is worth mentioning that in the beginning the SU(3) practitioners tended to make the assumption of the sextet $\mathbf{6}$ dominance over $\overline{\mathbf{1 5}}$. Under this hypothesis, one leads to $\mathcal{B}\left(\Lambda_{c}^{+} \rightarrow\right.$ $\left.p \pi^{0}\right) \sim 5 \times 10^{-4}[13,47]$, which exceeds the current experimental limit of $2.7 \times 10^{-4}$ [49]. Our dynamic calculation in [26] predicted $\mathcal{B}\left(\Lambda_{c}^{+} \rightarrow p \pi^{0}\right) \sim 1 \times 10^{-4}$. As far as the branching fraction is concerned, it is important to measure the mode $\Lambda_{c}^{+} \rightarrow n \pi^{+}$to distinguish our prediction from the

\footnotetext{
${ }^{12}$ Many early studies in the $S U(3)_{F}$ approach have overlooked the fact that charmed baryon decays are governed by several different partial-wave amplitudes that have distinct kinematic and dynamic effects.
}

SU(3) approach. As for decay asymmetries, while we agree on the sign and magnitude of $\alpha\left(\Xi^{0} K^{+}\right)$, we disagree on the sign of $\alpha$ in $\Lambda K^{+}$. Hopefully, these can be tested in the future. It is clear from Table VII that except for $\Xi_{c}^{+} \rightarrow \Sigma^{+} \bar{K}^{0}$, $\Xi^{0} \pi^{+}, \Xi^{0} K^{+}$, and $\Xi_{c}^{0} \rightarrow \Xi^{-} \pi^{+}, \Xi^{-} K^{+}$all the branching fractions of $\Xi_{c}^{+, 0}$ decays in this work and in the SU(3) approach are consistent with each other within a factor of 2 . Furthermore, we agree on the signs of decay asymmetries except $\Xi_{c}^{+} \rightarrow \Sigma^{+} \bar{K}^{0}$ and $\Xi_{c}^{+} \rightarrow \Xi^{0} K^{+}$. ${ }^{13}$ Notice that both approaches lead to $\mathcal{B}\left(\Xi_{c}^{0} \rightarrow \Xi^{-} \pi^{+}\right) \gg \mathcal{B}\left(\Xi_{c}^{+} \rightarrow \Xi^{0} \pi^{+}\right)$, contrary to the current data. Hence, it is of great importance to measure the branching fractions of them more accurately in order to test their underlying mechanism.

\section{Theoretical uncertainties}

In this subsection we discuss the major theoretical uncertainties one may encounter in this work.

\footnotetext{
${ }^{13}$ Those predictions of $\alpha$ with the uncertainty greater than the central value are not taken into account for comparison.
} 
TABLE VIII. Same as Table II except for $\Lambda_{c}^{+}$decays.

\begin{tabular}{lcccccrr}
\hline \hline Modes & $(c \bar{q})$ & $f_{1}\left(q_{\max }^{2}\right)$ & $f_{1}\left(m_{P}^{2}\right) / f_{1}\left(q_{\max }^{2}\right)$ & $f_{1}\left(m_{P}^{2}\right)$ & $g_{1}\left(q_{\max }^{2}\right)$ & $g_{1}\left(m_{P}^{2}\right) / g_{1}\left(q_{\max }^{2}\right)$ & $g_{1}\left(m_{P}^{2}\right)$ \\
\hline$\Lambda_{c}^{+} \rightarrow p \bar{K}^{0}$ & $(c \bar{d})$ & $-\frac{\sqrt{6}}{2} Y_{1}$ & 0.343423 & -0.371 & $-\frac{\sqrt{6}}{2} Y_{2}$ & 0.518518 & -0.488 \\
$\Lambda_{c}^{+} \rightarrow \Lambda \pi^{+}$ & $(c \bar{s})$ & $Y_{1}^{s}$ & 0.440793 & 0.419 & $Y_{2}^{s}$ & 0.590594 & 0.507 \\
$\Lambda_{c}^{+} \rightarrow p \pi^{0}$ & $(c \bar{d})$ & $-\frac{\sqrt{6}}{2} Y_{1}$ & 0.305365 & -0.330 & $-\frac{\sqrt{6}}{2} Y_{2}$ & 0.478571 & -0.450 \\
$\Lambda_{c}^{+} \rightarrow p \eta$ & $(c \bar{d}),(c \bar{s})$ & $-\frac{\sqrt{6}}{2} Y_{1}$ & 0.353139 & -0.382 & $-\frac{\sqrt{6}}{2} Y_{2}$ & 0.52837 & -0.497 \\
$\Lambda_{c}^{+} \rightarrow n \pi^{+}$ & $(c \bar{d})$ & $-\frac{\sqrt{6}}{2} Y_{1}$ & 0.306517 & -0.331 & $-\frac{\sqrt{6}}{2} Y_{2}$ & 0.479606 & -0.451 \\
$\Lambda_{c}^{+} \rightarrow \Lambda K^{+}$ & $(c \bar{s})$ & $Y_{1}^{s}$ & 0.494403 & 0.470 & $Y_{2}^{s}$ & 0.638728 & 0.549 \\
\hline \hline
\end{tabular}

(i) Wave functions in the MIT bag model. In the bag model the quark spatial wave function in the ground $1 S_{1 / 2}$ state has the expression

$$
\psi_{1 S_{1 / 2}}=\left(\begin{array}{c}
i u(r) \chi \\
v(r) \boldsymbol{\sigma} \cdot \hat{\mathbf{r}} \chi
\end{array}\right),
$$

where $u(r)$ and $v(r)$ are the large and small components of the quark wave function, respectively. In this work, we have employed the following bag parameters,

$$
\begin{aligned}
& m_{u}=m_{d}=0, \quad m_{s}=0.279 \mathrm{GeV}, \\
& m_{c}=1.551 \mathrm{GeV}, \quad R=5 \mathrm{GeV}^{-1},
\end{aligned}
$$

where $R$ is the radius of the bag. The uncertainties in the bag parameters affect the estimation of hadron matrix elements, form factors, and the strong couplings.

(ii) Form factors and Wilson parameters in factorizable amplitudes. The uncertainties in the factorizable amplitudes given in Eq. (15) arise from the Wilson parameters $a_{1,2}$ and the form factors $f_{1}\left(m_{P}^{2}\right)$ and $g_{1}\left(m_{P}^{2}\right)$. The measurement of $\Lambda_{c}^{+} \rightarrow p \phi$ allows us to fix $a_{2}$ to be $-0.45 \pm 0.05$ [26], which in turn implies that $a_{1}=1.26 \pm 0.02$. Form factors are first evaluated at zero recoil using the bag model. Their $q^{2}$ dependence is then determined based on the assumption of nearest pole dominance.

(iii) Nonfactorizable $S$-wave amplitude in current algebra. We have employed current algebra to evaluate $S$-wave amplitudes to circumvent the troublesome $1 / 2^{-}$ intermediate baryon resonances, which are not well understood in the quark model. Since current algebra is valid in the soft meson limit, it is natural to expect a correction of order $q^{2} / \Lambda_{\chi}^{2}$ where $\Lambda_{\chi} \sim 1 \mathrm{GeV}$ is a chiral symmetry breaking scale and $q^{2}$ is the c.m. three-momentum squared of the pseudoscalar meson produced in charmed baryon decays.

Among the antitriplet charmed baryon decays, $\Lambda_{c}^{+} \rightarrow p \phi$ is the only purely factorizable process. Also it is very difficult to quantify the errors from part (iii). Therefore, we focus on the uncertainties arising from the wave functions in the bag model. By varying the bag radius $R$ from $5.0 \mathrm{GeV}^{-1}$ (or $0.987 \mathrm{fm}$ ) to 4.8 and $5.2 \mathrm{GeV}^{-1}$, we obtain bag integrals slightly different from that given in Eqs. (D5) and (D14). This allows one to estimate the uncertainties in baryon matrix elements and the axial-vector form factors. Take $\Lambda_{c}^{+} \rightarrow \Xi^{0} K^{+}$and $\Xi_{c}^{0} \rightarrow \Sigma^{+} K^{-}$as examples for illustration as they proceed only through nonfactorizable diagrams. We obtain $\mathcal{B}\left(\Lambda_{c}^{+} \rightarrow \Xi^{0} K^{+}\right)=\left(0.73_{-0.15}^{+0.20}\right) \%$ and $\mathcal{B}\left(\Xi_{c}^{0} \rightarrow \Sigma^{+} K^{-}\right)=\left(0.46_{-0.10}^{+0.13}\right) \%$. Hence a slight change of the bag radius by $4 \%$ results in $(20-30) \%$ uncertainties in branching fractions.

\section{CONCLUSION}

In this work we have systematically studied the branching fractions and up-down decay asymmetries of CF and SCS decays of antitriplet charmed baryons. To estimate the nonfactorizable contributions, we work in the pole model for the $P$-wave amplitudes and current algebra for $S$-wave ones. Throughout the whole calculations, all the nonperturbative parameters, including form factors, baryon matrix elements, and axial-vector form factors are evaluated using the MIT bag model.

We draw some conclusions from our analysis.

(i) The long-standing puzzle with the branching fraction and decay asymmetry of $\Lambda_{c}^{+} \rightarrow \Xi^{0} K^{+}$is resolved by realizing that only the type-II $W$-exchange diagram contributes to this mode. We find that not only does the predicted rate agree with experiment, but also the decay asymmetry is consistent in sign and magnitude with the $S U(3)$ flavor approach. Hence, it is most likely that $\alpha\left(\Lambda_{c}^{+} \rightarrow \Xi^{0} K^{+}\right)$is large and positive.

(ii) In analog to $\Lambda_{c}^{+} \rightarrow \Xi^{0} K^{+}$, the CF mode $\Xi_{c}^{0} \rightarrow \Sigma^{+} K^{-}$ and the SCS decays $\Xi_{c}^{0} \rightarrow p K^{-}, \Sigma^{+} \pi^{-}$proceed only through type-II $W$ exchange. They are predicted to have large and positive decay asymmetries. This can be tested in the near future.

(iii) The predicted $\mathcal{B}\left(\Xi_{c}^{+} \rightarrow \Xi^{0} \pi^{+}\right)$agrees well with the measurement inferred from Belle and CLEO, while the calculated $\mathcal{B}\left(\Xi_{c}^{0} \rightarrow \Xi^{-} \pi^{+}\right)$is too large 
compared to the recent Belle measurement. We find $\mathcal{B}\left(\Xi_{c}^{0} \rightarrow \Xi^{-} \pi^{+}\right) \gg \mathcal{B}\left(\Xi_{c}^{+} \rightarrow \Xi^{0} \pi^{+}\right)$and conclude that these two modes cannot be simultaneously explained within the current-algebra framework for $S$-wave amplitudes. On-shell corrections to the current-algebra results are probably needed to circumvent the difficulty with $\Xi_{c}^{0} \rightarrow \Xi^{-} \pi^{+}$. More accurate measurements of them are called for to set the issue.

(iv) Owing to large cancellation between factorizable and nonfactorizable contributions for both $S$ - and $P$ wave amplitudes, we argue that the present theoretical predictions of $\Lambda_{c}^{+} \rightarrow n \pi^{+}$are unreliable. It is important to measure this SCS mode to understand its underlying mechanism.

(v) Although $\Xi_{c}^{0} \rightarrow \Sigma^{0} \bar{K}^{0}$ and $\Xi_{c}^{+} \rightarrow \Sigma^{+} \bar{K}^{0}$ are Cabibbofavored decays, their branching fractions are small especially for the former due to large destructive interference between factorizable and nonfactorizable amplitudes.

(vi) We have compared our results with the approach of SU(3) flavor symmetry. Excluding those predictions of $\alpha$ with the uncertainty greater than the central value, we find that both approaches agree on the signs of decay asymmetries except the decay modes, $\Xi_{c}^{+} \rightarrow \Sigma^{+} \bar{K}^{0}$ and $\Xi_{c}^{+} \rightarrow \Xi^{0} K^{+}$. We also agree on the hierarchy $\mathcal{B}\left(\Xi_{c}^{0} \rightarrow \Xi^{-} \pi^{+}\right) \gg$ $\mathcal{B}\left(\Xi_{c}^{+} \rightarrow \Xi^{0} \pi^{+}\right)$.

(vii) We have identified several major sources of theoretical uncertainties and given some crude estimation of errors on branching fractions provided that uncertainty arises from the MIT bag-model wave functions.

\section{ACKNOWLEDGMENTS}

We thank C. Q. Geng for discussion and for providing us the updated results in the flavor-SU(3) approach. This research was supported in part by the Ministry of Science and Technology of ROC under Grant No. 107-2119-M001-034. F.X. is supported by NSFC under Grants No. 11605076 and No. U1932104.

\section{APPENDIX A: BARYON WAVE FUNCTIONS}

Throughout this paper, we follow the convention in [26] for the wave functions of baryons with $S_{z}=1 / 2$,

$$
\begin{aligned}
p & =\frac{1}{\sqrt{3}}\left[u u d \chi_{S}+(13)+(23)\right], & n & =-\frac{1}{\sqrt{3}}\left[d d u \chi_{S}+(13)+(23)\right], \\
\Sigma^{+} & =-\frac{1}{\sqrt{3}}\left[u u s \chi_{S}+(13)+(23)\right], & \Sigma^{0} & =\frac{1}{\sqrt{6}}\left[(u d s+d u s) \chi_{S}+(13)+(23)\right], \\
\Xi^{0} & =\frac{1}{\sqrt{3}}\left[\operatorname{ssu} \chi_{S}+(13)+(23)\right], & \Xi^{-} & =\frac{1}{\sqrt{3}}\left[s s d \chi_{S}+(13)+(23)\right], \\
\Lambda & =-\frac{1}{\sqrt{6}}\left[(u d s-d u s) \chi_{A}+(13)+(23)\right], & \Lambda_{c}^{+} & =-\frac{1}{\sqrt{6}}\left[(u d c-d u c) \chi_{A}+(13)+(23)\right], \\
\Sigma_{c}^{+} & =\frac{1}{\sqrt{6}}\left[(u d c+d u c) \chi_{S}+(13)+(23)\right], & \Sigma_{c}^{0} & =\frac{1}{\sqrt{3}}\left[d d c \chi_{S}+(13)+(23)\right], \\
\Xi_{c}^{+} & =\frac{1}{\sqrt{6}}\left[(u s c-s u c) \chi_{A}+(13)+(23)\right], & \Xi_{c}^{0} & =\frac{1}{\sqrt{6}}\left[(d s c-s d c) \chi_{A}+(13)+(23)\right], \\
\Xi_{c}^{\prime+} & =\frac{1}{\sqrt{6}}\left[(u s c+s u c) \chi_{S}+(13)+(23)\right], & \Xi_{c}^{\prime 0} & =\frac{1}{\sqrt{6}}\left[(d s c+s d c) \chi_{S}+(13)+(23)\right], \\
\Omega_{c}^{0} & =\frac{1}{\sqrt{3}}\left[s s c \chi_{s}+(13)+(23)\right], & \Sigma^{-} & =\frac{1}{\sqrt{3}}\left[d d s \chi_{s}+(13)+(23)\right],
\end{aligned}
$$

where $a b c \chi_{S}=\left(2 a^{\uparrow} b^{\uparrow} c^{\downarrow}-a^{\uparrow} b^{\downarrow} c^{\uparrow}-a^{\downarrow} b^{\uparrow} c^{\uparrow}\right) / \sqrt{6}$ and $a b c \chi_{A}=\left(a^{\uparrow} b^{\downarrow} c^{\uparrow}-a^{\downarrow} b^{\uparrow} c^{\uparrow}\right) / \sqrt{2}$. There are two useful relations under the $U, V$, and $I$ spin,

$$
\begin{aligned}
& a b c \chi_{s}+\sqrt{3} a b c \chi_{A}=-\sqrt{\frac{2}{3}}\left(2 a^{\downarrow} b^{\uparrow} c^{\uparrow}-a^{\uparrow} b^{\uparrow} c^{\downarrow}-a^{\uparrow} b^{\downarrow} c^{\uparrow}\right), \\
& a b c \chi_{S}-\sqrt{3} a b c \chi_{A}=-\sqrt{\frac{2}{3}}\left(2 a^{\uparrow} b^{\downarrow} c^{\uparrow}-a^{\uparrow} b^{\uparrow} c^{\downarrow}-a^{\downarrow} b^{\uparrow} c^{\uparrow}\right) .
\end{aligned}
$$




\section{APPENDIX B: BARYONS UNDER $U, V$, AND I SPIN}

In practical calculations, we need to specify the behaviors of baryon wave functions under the isospin, $U$-spin, and $V$-spin ladder operators. Based on the wave functions given by Eq. (A1), we have the following relations,

$$
\begin{aligned}
U_{+}\left|\Sigma^{+}\right\rangle & =-|p\rangle, & U_{+}\left|\Xi^{-}\right\rangle & =-\left|\Sigma^{-}\right\rangle, \\
U_{+}\left|\Sigma^{0}\right\rangle & =\frac{\sqrt{2}}{2}|n\rangle, & U_{+}|\Lambda\rangle & =-\frac{\sqrt{6}}{2}|n\rangle, \\
U_{+}\left|\Xi^{0}\right\rangle & =-\frac{\sqrt{2}}{2}\left|\Sigma^{0}\right\rangle+\frac{\sqrt{6}}{2}|\Lambda\rangle, & U_{+}\left|\Xi_{c}^{+}\right\rangle & =-\left|\Lambda_{c}^{+}\right\rangle, \\
U_{-}\left|\Sigma^{0}\right\rangle & =-\frac{\sqrt{2}}{2}\left|\Xi^{0}\right\rangle, & U_{-}|\Lambda\rangle & =\frac{\sqrt{6}}{2}\left|\Xi^{0}\right\rangle, \\
U_{-}|n\rangle & =\frac{\sqrt{2}}{2}\left|\Sigma^{0}\right\rangle-\frac{\sqrt{6}}{2}|\Lambda\rangle, & U_{-}|p\rangle & =-\left|\Sigma^{+}\right\rangle,
\end{aligned}
$$

for $U$-spin ladder operators,

$$
\begin{aligned}
V_{+}\left|\Xi^{0}\right\rangle & =\left|\Sigma^{+}\right\rangle, & V_{+}\left|\Sigma^{-}\right\rangle & =-|n\rangle, \\
V_{+}|\Lambda\rangle & =-\frac{\sqrt{6}}{2}|p\rangle, & V_{+}\left|\Sigma^{0}\right\rangle & =-\frac{\sqrt{2}}{2}|p\rangle, \\
V_{+}\left|\Xi^{-}\right\rangle & =-\frac{\sqrt{2}}{2}\left|\Sigma^{0}\right\rangle-\frac{\sqrt{6}}{2}|\Lambda\rangle, & V_{+}\left|\Xi_{c}^{0}\right\rangle & =\left|\Lambda_{c}^{+}\right\rangle, \\
V_{-}|p\rangle & =-\frac{\sqrt{2}}{2}\left|\Sigma^{0}\right\rangle-\frac{\sqrt{6}}{2}|\Lambda\rangle, & V_{-}\left|\Sigma^{+}\right\rangle & =\left|\Xi^{0}\right\rangle,
\end{aligned}
$$

for $V$-spin ladder operators, and

$$
\begin{aligned}
& I_{+}|n\rangle=|p\rangle, \quad I_{+}\left|\Xi^{-}\right\rangle=\left|\Xi^{0}\right\rangle, \\
& I_{+}\left|\Sigma^{-}\right\rangle=\sqrt{2}\left|\Sigma^{0}\right\rangle, \quad I_{+}\left|\Sigma^{0}\right\rangle=-\sqrt{2}\left|\Sigma^{+}\right\rangle, \\
& I_{+}|\Lambda\rangle=0, \quad I_{+}\left|\Xi_{c}^{0}\right\rangle=\left|\Xi_{c}^{+}\right\rangle, \\
& I_{-}\left|\Xi_{c}^{+}\right\rangle=\left|\Xi_{c}^{0}\right\rangle, \quad I_{-}\left|\Sigma^{+}\right\rangle=-\sqrt{2}\left|\Sigma^{0}\right\rangle,
\end{aligned}
$$

for isospin ladder operators. Note that some of the relations may have signs different from the textbook due to our wave function convention. The ladder operators satisfy the commutator relations

$$
\left[U_{+}, U_{-}\right]=2 U_{3}, \quad\left[V_{+}, V_{-}\right]=2 V_{3}, \quad\left[I_{+}, I_{-}\right]=2 I_{3} .
$$

\section{APPENDIX C: FORM FACTORS FOR $\Lambda_{c}^{+}$DECAYS}

Form factors for $\Lambda_{c}^{+} \rightarrow \mathcal{B}$ transitions evaluated in the MIT bag model are shown in Table VIII. For $\Lambda_{c}^{+} \rightarrow p \eta_{8}$, we have assumed that form factors are dominated by the $(c \bar{d})$ quark content.

\section{APPENDIX D: HADRONIC MATRIX ELEMENTS AND AXIAL-VECTOR FORM FACTORS}

We use the MIT bag model to evaluate the baryon matrix elements and the axial-vector form factors (see, e.g., [34] for details).

\section{Baryon matrix elements}

The hadronic matrix elements $a_{\mathcal{B}^{\prime} \mathcal{B}}$ play an essential role both in $S$-wave and $P$-wave amplitudes. The general expressions are given by

$a_{\mathcal{B}^{\prime} \mathcal{B}} \equiv\left\langle\mathcal{B}^{\prime}\left|\mathcal{H}_{\mathrm{eff}}^{\mathrm{PC}}\right| \mathcal{B}\right\rangle=\left\{\begin{array}{l}\frac{G_{F}}{2 \sqrt{2}} V_{c s} V_{u d^{*}}^{*} c_{-}\left\langle\mathcal{B}^{\prime}\left|O_{-}\right| \mathcal{B}\right\rangle, \mathrm{CF} \\ \frac{G_{F}}{2 \sqrt{2}} V_{c q} V_{u q}^{*} c_{-}\left\langle\mathcal{B}^{\prime}\left|O_{-}^{q}\right| \mathcal{B}\right\rangle, \quad \mathrm{SCS}\end{array}\right.$

for CF and SCS processes, respectively, where $q=d, s$. Note that in SCS process there are in general two operators. For the definition of operators and Wilson coefficients, taking $\mathrm{CF}$ process as an example, we have $O_{-}=(\bar{s} c)(\bar{u} d)-(\bar{s} d)(\bar{u} c), \quad c_{-}=c_{1}-c_{2}$ and then we have the relation $c_{+} O_{+}+c_{-} O_{-}=2\left(c_{1} O_{1}+c_{2} O_{2}\right)$. The matrix element of $O_{+}$vanishes since this operator is symmetric in color indices. Below, we show the results of $\left\langle\mathcal{B}^{\prime}\left|O_{-}^{(q)}\right| \mathcal{B}\right\rangle$ in the MIT bag model.

The relevant matrix elements for Cabibbo-favored processes are

$$
\begin{aligned}
\left\langle\Sigma^{+}\left|O_{-}\right| \Lambda_{c}^{+}\right\rangle & =-\frac{2 \sqrt{6}}{3}\left(X_{1}+3 X_{2}\right)(4 \pi), \\
\left\langle\Xi^{0}\left|O_{-}\right| \Xi_{c}^{0}\right\rangle & =\frac{2 \sqrt{6}}{3}\left(X_{1}-3 X_{2}\right)(4 \pi), \\
\left\langle\Xi^{0}\left|O_{-}\right| \Xi_{c}^{\prime 0}\right\rangle & =-\frac{2 \sqrt{2}}{3}\left(X_{1}+9 X_{2}\right)(4 \pi), \\
\left\langle\Sigma^{0}\left|O_{-}\right| \Sigma_{c}^{0}\right\rangle & =-\frac{2 \sqrt{2}}{3}\left(X_{1}-9 X_{2}\right)(4 \pi), \\
\left\langle\Sigma^{+}\left|O_{-}\right| \Sigma_{c}^{+}\right\rangle & =\frac{2 \sqrt{2}}{3}\left(X_{1}-9 X_{2}\right)(4 \pi), \\
\left\langle\Lambda\left|O_{-}\right| \Sigma_{c}^{0}\right\rangle & =-\frac{2 \sqrt{6}}{3}\left(X_{1}+3 X_{2}\right)(4 \pi),
\end{aligned}
$$

while the nonvanishing matrix elements for SCS decays are given by 


$$
\begin{aligned}
\left\langle\Sigma^{0}\left|O_{-}^{d}\right| \Xi_{c}^{0}\right\rangle & =-\frac{4 \sqrt{3}}{3} X_{1}^{d}(4 \pi), & \left\langle\Sigma^{0}\left|O_{-}^{d}\right| \Xi_{c}^{\prime 0}\right\rangle & =\frac{4}{3} X_{1}^{d}(4 \pi), \\
\left\langle\Lambda\left|O_{-}^{d}\right| \Xi_{c}^{0}\right\rangle & =-4 X_{2}^{d}(4 \pi), & \left\langle\Lambda\left|O_{-}^{d}\right| \Xi_{c}^{\prime 0}\right\rangle & =-4 \sqrt{3} X_{2}^{d}(4 \pi), \\
\left\langle p\left|O_{-}^{d}\right| \Sigma_{c}^{+}\right\rangle & =-\frac{2 \sqrt{2}}{3}\left(X_{1}^{d}-9 X_{2}^{d}\right)(4 \pi), & \left\langle p\left|O_{-}^{d}\right| \Lambda_{c}^{+}\right\rangle & =\frac{2 \sqrt{6}}{3}\left(X_{1}^{d}+3 X_{2}^{d}\right)(4 \pi), \\
\left\langle n\left|O_{-}^{d}\right| \Sigma_{c}^{0}\right\rangle & =\frac{4}{3}\left(X_{1}^{d}+9 X_{2}^{d}\right)(4 \pi), & \left\langle\Sigma^{0}\left|O_{-}^{s}\right| \Xi_{c}^{\prime 0}\right\rangle & =-\frac{2}{3}\left(X_{1}^{s}-9 X_{2}^{s}\right)(4 \pi), \\
\left\langle\Lambda\left|O_{-}^{s}\right| \Xi_{c}^{0}\right\rangle & =-2\left(X_{1}^{s}-X_{2}^{s}\right)(4 \pi), & \left\langle\Lambda\left|O_{-}^{s}\right| \Xi_{c}^{\prime 0}\right\rangle & =-\frac{2 \sqrt{3}}{3}\left(X_{1}^{s}+3 X_{2}^{s}\right)(4 \pi), \\
\left\langle\Xi^{0}\left|O_{-}^{s}\right| \Omega_{c}^{0}\right\rangle & =-\frac{4}{3}\left(X_{1}^{s}+9 X_{2}^{s}\right)(4 \pi), & \left\langle\Sigma^{+}\left|O_{-}^{s}\right| \Xi_{c}^{+}\right\rangle & =\frac{2 \sqrt{6}}{3}\left(X_{1}^{s}+3 X_{2}^{s}\right)(4 \pi), \\
\left\langle\Sigma^{+}\left|O_{-}^{s}\right| \Xi_{c}^{\prime+}\right\rangle & =\frac{2 \sqrt{2}}{3}\left(X_{1}^{s}-9 X_{2}^{s}\right)(4 \pi), & \left\langle\Sigma^{0}\left|O_{-}^{s}\right| \Xi_{c}^{0}\right\rangle & =-\frac{2 \sqrt{3}}{3}\left(X_{1}^{s}+3 X_{2}^{s}\right)(4 \pi),
\end{aligned}
$$

where we have introduced the bag integrals

$$
\begin{array}{ll}
X_{1}=\int_{0}^{R} r^{2} d r\left(u_{s} v_{u}-v_{s} u_{u}\right)\left(u_{c} v_{d}-v_{c} u_{d}\right), & X_{2}=\int_{0}^{R} r^{2} d r\left(u_{s} u_{u}+v_{s} v_{u}\right)\left(u_{c} u_{d}+v_{c} v_{d}\right), \\
X_{1}^{q}=\int_{0}^{R} r^{2} d r\left(u_{q} v_{u}-v_{q} u_{u}\right)\left(u_{q} v_{c}-v_{q} u_{c}\right), & X_{2}^{q}=\int_{0}^{R} r^{2} d r\left(u_{q} v_{u}+v_{q} u_{u}\right)\left(u_{q} v_{c}+v_{q} u_{c}\right),
\end{array}
$$

with $q=d$, s. Numerically, we obtain

$$
\begin{aligned}
& X_{1}^{d}=0, \quad X_{2}^{d}=1.60 \times 10^{-4}, \quad X_{1}^{s}=2.60 \times 10^{-6}, \\
& X_{2}^{s}=1.96 \times 10^{-4}, \quad X_{1}=3.56 \times 10^{-6}, \quad X_{2}=1.74 \times 10^{-4} .
\end{aligned}
$$

\section{Axial-vector form factors}

In the MIT bag model the axial form factor in the static limit can be expressed as

$$
g_{\mathcal{B}^{\prime} \mathcal{B}}^{A(P)}=\left\langle\mathcal{B}^{\prime} \uparrow\left|b_{q_{1}}^{\dagger} b_{q_{2}} \sigma_{z}\right| \mathcal{B} \uparrow\right\rangle \int d^{3} \boldsymbol{r}\left(u_{q_{1}} u_{q_{2}}-\frac{1}{3} v_{q_{1}} v_{q_{2}}\right) .
$$

Based on Eq. (D6), the axial-vector form factors related to CF processes are ${ }^{14}$

$$
\begin{gathered}
g_{\Lambda_{c}^{+} \Xi_{c}^{+}}^{A\left(\bar{K}^{0}\right)}=g_{\Xi_{c}^{0} \Xi_{c}^{+}}^{A\left(\pi^{+}\right)}=g_{\Xi_{c}^{0} \Xi_{c}^{0}}^{A\left(\pi^{0}\right)}=g_{\Xi_{c}^{0} \Xi_{c}^{0}}^{A\left(\eta_{8}\right)}=0, \\
g_{\Xi_{c}^{\prime \prime} \Xi_{c}^{+}}^{A\left(\pi^{+}\right)}=\sqrt{3} g_{\Xi^{-} \Xi^{0}}^{A\left(\pi^{+}\right)}=-2 g_{\Xi_{c}^{\prime} \Xi_{c}^{0}}^{A\left(\pi^{0}\right)}=2 \sqrt{3} g_{\Xi^{0} \Xi^{0}}^{A\left(\pi^{0}\right)}=\frac{2}{\sqrt{3}} g_{\Xi_{c}^{\prime 0} \Xi_{c}^{0}}^{A\left(\eta_{8}\right)} \\
=\frac{2}{3} g_{\Xi^{0} \Xi^{0}}^{A\left(\eta_{8}\right)}=g_{\Lambda \Lambda}^{A\left(\eta_{8}\right)}=-\frac{\sqrt{3}}{3}\left(4 \pi Z_{1}\right), \\
g_{\Sigma_{c}^{+} \Xi_{c}^{+}}^{A\left(\bar{K}^{0}\right)}=\frac{\sqrt{2}}{2} g_{\Sigma_{c}^{0} \Xi_{c}^{0}}^{A\left(\bar{K}^{0}\right)}=\sqrt{2} g_{\Lambda \Xi^{0}}^{A\left(\bar{K}^{0}\right)}=-\frac{\sqrt{6}}{5} g_{\Sigma^{0} \Xi^{0}}^{A\left(\bar{K}^{0}\right)}=\frac{\sqrt{3}}{5} g_{\Sigma^{+} \Xi^{0}}^{A\left(K^{-}\right)}=\frac{\sqrt{3}}{3}\left(4 \pi Z_{2}\right),
\end{gathered}
$$

and

$$
g_{\Xi_{c}^{+} \Xi_{c}^{+}}^{A\left(\eta_{8}\right)}=g_{\Sigma^{0} \Lambda}^{A\left(\eta_{8}\right)}=g_{\Lambda \Sigma^{0}}^{A\left(\eta_{8}\right)}=g_{\Lambda \Lambda}^{A\left(\pi^{0}\right)}=g_{\Sigma^{0} \Sigma^{0}}^{A\left(\pi^{0}\right)}=0
$$

\footnotetext{
${ }^{14}$ Recall that the axial-vector current is $\left(\bar{u} \gamma_{\mu} \gamma_{5} u-\bar{d} \gamma_{\mu} \gamma_{5} d\right) / 2$ for $\pi^{0}$ and $\left(\bar{u} \gamma_{\mu} \gamma_{5} u+\bar{d} \gamma_{\mu} \gamma_{5} d-2 \bar{s} \gamma_{\mu} \gamma_{5} s\right) /(2 \sqrt{3})$ for $\eta_{8}$ in our convention.
} 


$$
\begin{gathered}
g_{\Lambda \Sigma^{0}}^{A\left(\pi^{0}\right)}=g_{\Sigma^{0} \Lambda}^{A\left(\pi^{0}\right)}=g_{\Sigma^{0} \Sigma^{0}}^{A\left(\eta_{8}\right)}=g_{\Sigma^{+} \Sigma^{+}}^{A\left(\eta_{8}\right)}=-g_{\Lambda \Lambda}^{A\left(\eta_{8}\right)}=-\frac{2 \sqrt{3}}{3} g_{\Xi_{c}^{\prime+} \Xi_{c}^{+}}^{A\left(\eta_{8}\right)}=\frac{\sqrt{3}}{2} g_{\Sigma^{+} \Sigma^{+}}^{A\left(\pi^{0}\right)}=\frac{\sqrt{2}}{2} g_{\Lambda \Sigma^{+}}^{A\left(\pi^{+}\right)}, \\
=-\frac{\sqrt{6}}{4} g_{\Sigma^{0} \Sigma^{+}}^{A\left(\pi^{+}\right)}=\frac{\sqrt{6}}{4} g_{\Sigma^{-} \Sigma^{0}}^{A\left(\pi^{+}\right)}=\frac{\sqrt{2}}{2} g_{\Sigma^{-} \Lambda}^{A\left(\pi^{+}\right)}=-\frac{\sqrt{6}}{4} g_{\Sigma^{+} \Sigma^{0}}^{A\left(\pi^{-}\right)}=\frac{\sqrt{2}}{2} g_{\Sigma^{+} \Lambda}^{A\left(\pi^{-}\right)}=\frac{\sqrt{3}}{3}\left(4 \pi Z_{1}\right), \\
g_{p \Sigma^{+}}^{A\left(\bar{K}^{0}\right)}=-\frac{\sqrt{6}}{6} g_{\Omega_{c}^{0} \Xi_{c}^{+}}^{A\left(K^{+}\right)}=-\frac{\sqrt{6}}{6} g_{\Omega_{c}^{0} \Xi_{c}^{0}}^{A\left(K^{0}\right)}=-\frac{\sqrt{2}}{5} g_{\Xi^{0} \Sigma^{0}}^{A\left(K^{0}\right)}=\frac{\sqrt{6}}{3} g_{\Xi^{0} \Lambda}^{A\left(K^{0}\right)} \\
=-\frac{\sqrt{2}}{5} g_{\Xi^{-} \Sigma^{0}}^{A\left(K^{+}\right)}=-\frac{\sqrt{6}}{3} g_{\Xi^{-} \Lambda}^{A\left(K^{+}\right)}=\sqrt{2} g_{p \Sigma^{0}}^{A\left(K^{-}\right)}=-\frac{\sqrt{6}}{9} g_{p \Lambda}^{A\left(K^{-}\right)} \\
=-\frac{\sqrt{6}}{9} g_{n \Lambda}^{A\left(\bar{K}^{0}\right)}=-\sqrt{2} g_{n \Sigma^{0}}^{A\left(\bar{K}^{0}\right)}=\frac{1}{5} g_{\Xi^{0} \Sigma^{+}}^{A\left(K^{+}\right)}=\frac{1}{3}\left(4 \pi Z_{2}\right),
\end{gathered}
$$

for SCS processes, where the auxiliary parameters are introduced,

$$
Z_{1}=\int r^{2} d r\left(u_{u}^{2}-\frac{1}{3} v_{u}^{2}\right), \quad Z_{2}=\int r^{2} d r\left(u_{u} u_{s}-\frac{1}{3} v_{u} v_{s}\right)
$$

in the bag model. The numerical results are

$$
(4 \pi) Z_{1}=0.65, \quad(4 \pi) Z_{2}=0.71
$$

[1] R. Aaij et al. (LHCb Collaboration), Phys. Rev. Lett. 122, 211803 (2019).

[2] R. Aaij et al. (LHCb Collaboration), Phys. Rev. Lett. 119, 112001 (2017).

[3] R. Aaij et al. (LHCb Collaboration), Phys. Rev. Lett. 121, 052002 (2018).

[4] R. Aaij et al. (LHCb Collaboration), Phys. Rev. Lett. 121, 162002 (2018).

[5] A. Zupanc et al. (Belle Collaboration), Phys. Rev. Lett. 113, 042002 (2014).

[6] M. Ablikim et al. (BESIII Collaboration), Phys. Rev. Lett. 116, 052001 (2016).

[7] M. Tanabashi et al. (Particle Data Group), Phys. Rev. D 98, 030001 (2018).

[8] M. Ablikim et al. (BESIII Collaboration), Phys. Rev. D 95, 111102 (2017).

[9] Y. B. Li et al. (Belle Collaboration), Phys. Rev. Lett. 122, 082001 (2019).

[10] Y. B. Li et al. (Belle Collaboration), Phys. Rev. D 100, 031101 (2019).

[11] K. W. Edwards et al. (CLEO Collaboration), Phys. Lett. B 373, 261 (1996).

[12] R. Aaij et al. (LHCb Collaboration), Phys. Rev. D 100, 032001 (2019).

[13] C. Q. Geng, Y. K. Hsiao, C. W. Liu, and T. H. Tsai, Phys. Rev. D 97, 073006 (2018).

[14] C. Q. Geng, Y. K. Hsiao, C. W. Liu, and T. H. Tsai, Eur. Phys. J. C 78, 593 (2018).
[15] C. Q. Geng, C. W. Liu, and T. H. Tsai, Phys. Lett. B 794, 19 (2019).

[16] H. J. Zhao, Y. K. Hsiao, and Y. Yao, arXiv:1811.07265.

[17] Z. X. Zhao, Chin. Phys. C 42, 093101 (2018).

[18] C. P. Jia, D. Wang, and F. S. Yu, arXiv:1910.00876.

[19] L. L. Chau, H. Y. Cheng, and B. Tseng, Phys. Rev. D 54, 2132 (1996).

[20] J. G. Korner and M. Kramer, Z. Phys. C 55, 659 (1992).

[21] M. A. Ivanov, J. G. Korner, V. E. Lyubovitskij, and A. G. Rusetsky, Phys. Rev. D 57, 5632 (1998).

[22] Q. P. Xu and A. N. Kamal, Phys. Rev. D 46, 270 (1992).

[23] H. Y. Cheng and B. Tseng, Phys. Rev. D 48, 4188 (1993).

[24] P. Żenczykowski, Phys. Rev. D 50, 5787 (1994).

[25] K. K. Sharma and R. C. Verma, Eur. Phys. J. C 7, 217 (1999).

[26] H. Y. Cheng, X. W. Kang, and F. Xu, Phys. Rev. D 97, 074028 (2018).

[27] M. Bishai et al. (CLEO Collaboration), Phys. Lett. B 350, 256 (1995).

[28] P. Żenczykowski, Phys. Rev. D 50, 402 (1994).

[29] A. Datta, arXiv:hep-ph/9504428.

[30] M. Ablikim et al. (BESIII Collaboration), Phys. Rev. D 100, 072004 (2019).

[31] G. Buchalla, A. J. Buras, and M. E. Lautenbacher, Rev. Mod. Phys. 68, 1125 (1996).

[32] T. Feldmann, P. Kroll, and B. Stech, Phys. Lett. B 449, 339 (1999); Phys. Rev. D 58, 114006 (1998). 
[33] R. Perez-Marcial, R. Huerta, A. Garcia, and M. Avila-Aoki, Phys. Rev. D 40, 2955 (1989); 44, 2203(E) (1991).

[34] H. Y. Cheng and B. Tseng, Phys. Rev. D 46, 1042 (1992); 55, 1697(E) (1997).

[35] R. N. Faustov and V. O. Galkin, Eur. Phys. J. C 79, 695 (2019).

[36] A. Chodos, R. L. Jaffe, K. Johnson, and C. B. Thorn, Phys. Rev. D 10, 2599 (1974); T. A. DeGrand, R. L. Jaffe, K. Johnson, and J. E. Kiskis, Phys. Rev. D 12, 2060 (1975).

[37] F. E. Close, An Introduction to Quarks and Partons (Academic Press, London, 1979).

[38] D. Fakirov and B. Stech, Nucl. Phys. B133, 315 (1978).

[39] T. Gutsche, M. A. Ivanov, J. G. Krner, V. E. Lyubovitskij, and P. Santorelli, Phys. Rev. D 90, 114033 (2014); 94, 059902(E) (2016).

[40] M. Ablikim et al. (BESIII Collaboration), Phys. Lett. B 783, 200 (2018).

[41] S. Chan et al. (CLEO Collaboration), Phys. Rev. D 63, 111102 (2001).
[42] M. J. Savage and R. P. Springer, Phys. Rev. D 42, 1527 (1990); M. J. Savage, Phys. Lett. B 257, 414 (1991).

[43] S. M. Sheikholeslami, M. P. Khanna, and R. C. Verma, Phys. Rev. D 43, 170 (1991); R. C. Verma and M. P. Khanna, Phys. Rev. D 53, 3723 (1996).

[44] L. L. Chau, H. Y. Cheng, and B. Tseng, Phys. Rev. D 54, 2132 (1996).

[45] Y. Kohara, Phys. Rev. D 44, 2799 (1991).

[46] K. K. Sharma and R. C. Verma, Phys. Rev. D 55, 7067 (1997).

[47] C. D. Lu, W. Wang, and F. S. Yu, Phys. Rev. D 93, 056008 (2016).

[48] C. Q. Geng (privite communication).

[49] M. Ablikim et al. (BESIII Collaboration), Phys. Rev. D 95, 111102 (2017).

Correction: The fifth lines in both Tables IV (columns 5, 6, 7, and 9) and VII (column 2) contained errors and have been fixed. 